\title{
Certification of New Standard Reference Material 2806b Medium Test Dust in Hydraulic Fluid
}

\author{
Robert Fletcher, Nicholas Ritchie, David Bright, James Filliben, and William Guthrie \\ National Institute of Standards and Technology, \\ Gaithersburg, MD, 20899 \\ robert.fletcher@nist.gov \\ nicholas.ritchie@nist.gov \\ david.bright@nist.gov \\ james.filliben@nist.gov \\ william.guthrie@nist.gov
}

\begin{abstract}
A new material has been certified to become Standard Reference Material (SRM) 2806b - Medium Test Dust in Hydraulic Fluid. SRM 2806b consists of trace polydisperse, irregularly shaped mineral dust particles suspended in hydraulic fluid. The certified values of SRM 2806b are the projected area circular-equivalent diameters of the collected dust particles from the hydraulic fluid. The dimensional measurements were determined from the area of the collected dust particles using images obtained from automated scanning electron microscopy (SEM) followed by image analysis. An automated SEM and an automated image analysis software allowed the processing of over 29 million particles. The dimensional calibration of the SEM images (actual length per pixel and thus the actual projected diameters) are traceable to the NIST Line Scale Interferometer (LSI) through a NIST calibrated Geller MRS-4XY pitch standard. The certified diameters are correlated with the numeric concentration of particles greater than each diameter, referred to as the cumulative number size distribution. SRM 2806b is intended to be used to calibrate liquid-borne optical particle counters in conjunction with the reference method ISO 11171:2010.
\end{abstract}

Key words: calibration material; diameter; image analysis; liquid optical particle counter; particle; reference; scanning electron microscopy (SEM); SRM; standard.

Accepted: October 3, 2016

Published: December 19, 2016

https://doi.org/10.6028/jres.121.025

\section{Introduction}

SRM 2806b is intended for use as a reference material for calibrating liquid particle sizing instruments, especially optical particle counters. The Fluid Power Industry estimates that about $80 \%$ of all hydraulic failures are due to particulate contamination in the hydraulic fluid. Equipment failure is costly in terms of repairs, lost productivity, safety, and waste generation. Particle counters are routinely used to monitor fluid cleanliness in operating systems and to assess cleanliness of equipment coming off production lines as well as of individual components. SRM 2806 is a particle counter calibrant for liquid suspensions and a required reference material for ISO 11171:2010 Hydraulic fluid power - Calibration of automatic particle counters for liquids [1]. Industries impacted by SRM 2806 include hydraulics industry, aerospace, automotive, ship, petroleum and lubricant, gas, power generation, filter manufacturers and the military.

SRM 2806b - Medium Test Dust in Hydraulic Fluid is composed of MIL-PRF-5606 hydraulic fluid (NYCO Synthetic Lubricants, Paris, France, http://everyspec.com/MIL-PRF/MIL-PRF-00010009999/MIL-PRF-5606H_5993) with an added trace amount of SAE 5-80 Medium Test Dust (Particle Technology Inc., Arden Hills, MN) [2]. The polydisperse medium test dust came from the same batch of dry dust, 4390C, that was used to make SRM 2806, SRM 2806a, RM 8631 and RM 8631a. The SRM was 
manufactured by the International Filter Testing Service (IFTS) ${ }^{1}$ in France (July 26, 2011) in an approximately $200 \mathrm{~L}$ batch with a specified dust level, and split into $4000.5 \mathrm{~L}$ bottles, each bottle sequentially labeled to denote its place in the production sequence.

The certified properties of SRM 2806b are in terms of the projected area diameters of the collected dust particles from the hydraulic fluid. The diameters are made traceable to the NIST Line Scale Interferometer (LSI) through a NIST calibration of a Geller MRS-4XY pitch standard [3, 4]. The certified diameters are correlated with the numeric concentration of particles greater than each diameter, referred to as the cumulative number size distribution. Users of SRM 2806b calibrate their optical particle counters using the cumulative size distribution in conjunction with ISO 11171:2010. There is a unique particle diameter relation for each point on the cumulative size distribution. The certified values were determined by measurement with an automated scanning electron microscopy (SEM) coupled with advanced image analysis. Over 40 thousand images and 29 million individual particles were analyzed. The major steps taken to determine the particle size distribution for certification of SRM 2806b were:

(1) IFTS produced 400 bottles of SAE 5-80 medium test dust in hydraulic fluid material.

(2) An experimental design was developed through the NIST Statistical Engineering Division to determine the number of bottles to be analyzed.

(3) NIST designed multiple statistical tests coded in DATAPLOT [5, 6] to assure the production process of SRM 2806b produced a high quality material.

(4) Material homogeneity measurements were made with laser-based extinction particle counters and statistical tests designed by NIST. The homogeneity across the batch of 400 bottles was first determined through measurements by the material manufacturer. There were 40 bottles sampled by IFTS (10\% of the total number of bottles) in a randomized, blocked and balanced manner and analyzed by an optical particle counter. Data was provided to NIST for analysis. NIST then sampled 8 bottles for optical particle analysis. Results for IFTS and NIST were compared in Table 1.

Table 1. Comparison of the particle concentration variability specified at select particle diameters in terms of relative standard deviation measured by IFTS and NIST for candidate test material

\begin{tabular}{ccccc}
\hline $\begin{array}{c}\text { Diameter } \\
(\mu \mathrm{m})\end{array}$ & $\begin{array}{c}\text { IFTS } \\
\text { Mean } \\
\text { Value } \\
(1 / \mathrm{mL})\end{array}$ & $\begin{array}{c}\text { IFTS } \\
\text { RSD } \\
(\mathrm{n}=40)\end{array}$ & $\begin{array}{c}\text { NIST } \\
\text { Mean } \\
\text { Value } \\
(1 / \mathrm{mL})\end{array}$ & $\begin{array}{c}\text { NIST } \\
\text { RSD } \\
(\mathrm{n}=24)\end{array}$ \\
\hline$>4$ & 9807 & 0.0092 & 7338 & 0.0097 \\
$>6$ & 3300 & 0.012 & 2869 & 0.012 \\
$>10$ & 799 & 0.0185 & 696 & 0.015 \\
$>12$ & & & 401 & 0.015 \\
$>14$ & 275 & 0.018 & 229 & 0.016 \\
$>20$ & & & 53.48 & 0.022 \\
$>25$ & 30.18 & 0.034 & 25.25 & 0.028 \\
$>30$ & 15.025 & 0.044 & 13.34 & 0.038 \\
\hline
\end{tabular}

The NIST-developed statistical software package mentioned above was designed to verify that there was no systematic bias or variation in the bottled material. These tests that will be described in a future publication, were used on both data sets and each data set showed the same degree of homogeneity, material variability and lack of systematic bias. Difference in mean values (Table 1) most likely reflect a difference in sensor calibration between the two institutions.

${ }^{1}$ Certain commercial equipment, instruments, or materials are identified in this report to specify adequately the experimental procedure. Such identification does not imply recommendation or endorsement by the NIST, nor does it imply that the materials or equipment identified are necessarily the best available for the purpose. 
(5) A separate eight bottles were chosen randomly that spanned the production cycle of the material for microscopy.

(6) The mineral dust was separated from the hydraulic fluid by quantitative filtration onto polycarbonate filters.

(7) The filters were cleaned, dried and Au coated, then scanning electron microscope images were taken of selected regions of the filter at 4 different microscope magnifications.

(8) SEM images were analyzed to obtain particle areas of the individual particles.

(9) The particle areas were made traceable to the SI unit of length (meter) through a NIST-calibrated Geller MRS-4XY (Geller MicroAnalytical Laboratory, Inc., Topsfield, MA) scanning electron microscope $10 \times$ to $50000 \times$ pitch type of length or scale standard. The Geller standard was imaged concurrently with and under the same conditions as the particle images.

(10) Particle projected area (pixel count) was determined for all particles in the images.

(11) Particle area and number data were converted to particle size distributions using pixel to micrometer conversion factors determined by measuring images of the Geller length standard.

(12) Measurement uncertainties were determined in collaboration with the NIST Statistical Engineering Division.

We present details of the automated SEM image collection and image analysis that enabled a large number of particles to be characterized for the certification of this SRM.

\section{Experimental Procedure}

Characterization was conducted using two techniques: (1) automatic optical particle counting using an HRLD 600 sensor equipped with an auto sampler operating in volumetric mode (Beckman Coulter, Indianapolis, IN); and (2) automated microscopy using a field emission scanning electron microscope (TESCAN MIRA3, TESCAN, Brno, Czech Republic) that has been customized by NIST for rapid analysis of particles. We performed optical particle counting measurements to determine the particle size distribution and to test the hypothesis that all bottles contain the same particle population. Once a batch of prospective material was shown to be homogeneous, a separate set of bottles were selected to determine the particle size distribution by scanning electron microscopy and image analysis.

We analyzed bottles chosen by the following statistical method: bottles were taken from the global collection of 400 bottles, using a selection criteria to sample the material over the complete production sequence, but in a randomized manner, that was blocked and balanced to protect against possible systematic variations due to experimental conditions, such as bottle filling and/or mixing inhomogeneity. We chose the 8 bottles at random such that 2 each were taken from the 4 quartiles of the 400 bottle set. One of the bottles supplied 2 filters, one bottle supplied 4 filters and the remaining 6 bottles provided 1 filter giving a total of 12 filters for SEM imaging and analysis. The bottles were identified in the order of their creation using numbers from 1 to 400 , letters correspond to fill nozzle location. The bottle numbers used in the analysis are 314B (4 times), 211C (twice), 335B, 079B, 229D, 187C, 011C and 195C.

Because the mineral particles settle out of the hydraulic fluid, all bottles of candidate SRM 2806b were first shaken vigorously for 1 minute on a commercial paint shaker, then sonicated for 1 min using a sonic bath with power $>5 \mathrm{~W} / \mathrm{cm}^{2}$, then shaken again for $3 \mathrm{~min}$ to $5 \mathrm{~min}$, and analyzed or filtered immediately. Approximately $30 \mathrm{~mL}$ to $50 \mathrm{~mL}$ of hydraulic fluid was filtered using $90 \mathrm{~mm}$ diameter, $0.4 \mu \mathrm{m}$ pore size polycarbonate filters. The volume of hydraulic fluid sampled was determined by mass difference: each sample bottle was weighed before and after filtration on a Mettler Toledo balance, model XS2002S (Mettler-Toledo, LLC, Columbus, OH). The density of the hydraulic fluid was measured over the relevant temperature range by S. Sheckels, PML, NIST Fluid Metrology Laboratory, using a Rudolph Research Density Meter (Rudolph Research Analytical, Hackettstown, NJ) and found to be $(0.870968+/-0.000011)$ $\mathrm{g} / \mathrm{mL}$ at $21^{\circ} \mathrm{C}$. Measurements were compared to a reference hydrometer at $22.36^{\circ} \mathrm{C}$ giving a small difference of $8.04 \times 10^{-5} \mathrm{~g} / \mathrm{mL}$, which is an insignificant difference for measurement purposes. The filtered samples (filter + filtrate) were washed 3 to 4 times each with clean, doubly filtered heptane solvent to 
remove the oil residue. The solvent contained approximately $20 \mu \mathrm{L} / 500 \mathrm{~mL}$ of Stadis 450 (Innospec Limited, Ellesmere Port, UK), an additive to reduce static electricity effects in the dielectric solvent. Rather than a glass filter apparatus, a metal filter apparatus was used to reduce particles sticking to its walls. Before and after filtering the hydraulic fluid, heptane solvent was run through separate clean filters to detect any contamination due to the filtration device or to the solvent. These filters were processed in the same way as the sample filters. The filters were placed in vacuum at $28 \mathrm{kPa}$ (210 torr) for over $48 \mathrm{~h}$ to remove any volatile compounds. Filters were coated with $15 \mathrm{~nm}$ thick layer of gold by an Ar ion sputter coater (Cressington 208HR sputter coater, Cressington Scientific Instruments Ltd., Watford, England), mounted on a metal stage and imaged using the TESCAN MIRA3 automated SEM. A typical SEM image of the mineral dust at $0.25 \mathrm{~mm}$ field-of-view is shown in Fig. 1.

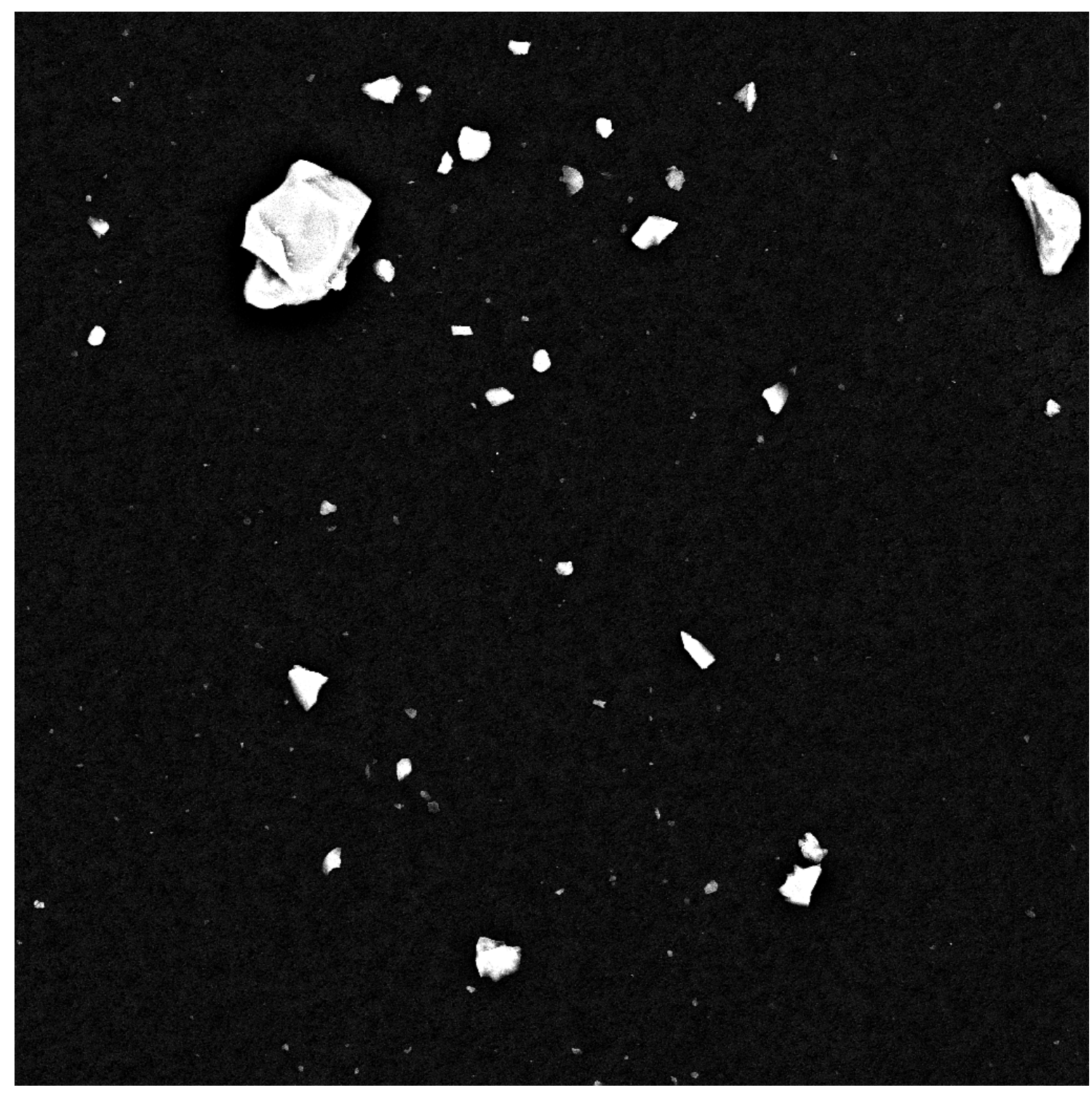

Fig. 1. Backscatter SEM image of medium test dust. Field-of-view is $250 \mu \mathrm{m}$. 


\section{Geller Scale Calibration Standard}

A Geller MRS-4XY scanning electron microscope $10 \times$ to 50,000 $\times$ pitch standard was made traceable to the SI by means of the NIST Line Scale Interferometer (LSI) [3, 4]. W. B. Penzes, NIST Precision Engineering Division (683) did the measurements. An electron image of the pitch standard is shown in Fig. 2. The Geller standard has suitable patterns to calibrate length or area in the images, i.e., to convert particle area in pixels to area in $\mu^{2}$. We calibrated both the horizontal and vertical dimensions.

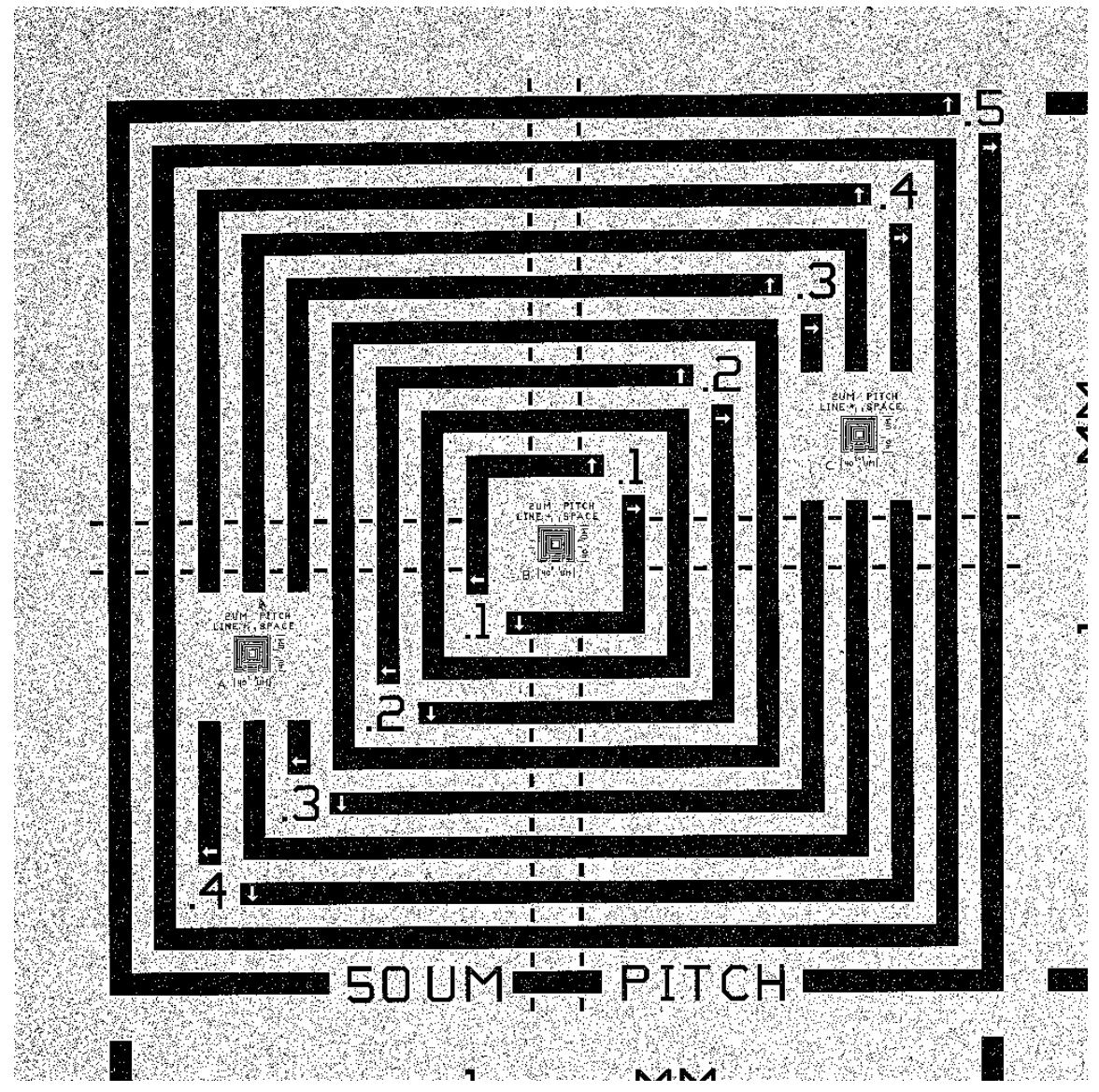

Fig. 2. High-resolution image of the Geller standard - $8192 \times 8192$ pixels, $66 \mathrm{MB}$. The region calibrated was over the $50 \mu \mathrm{m}$ spacing both horizontally and vertically.

\section{SEM Imaging}

The Au-coated $90 \mathrm{~mm}$ diameter filters were mounted on a circular metal stub that used a copper metal ring to stretch and hold the filter in place while providing a flat, electrically conductive pathway without gluing the filter in place. The TESCAN MIRA3 scanning electron microscope collected equal number of backscattered and secondary electron images of the entire filters. The analysis area was identified by three fiducial points around the perimeter of the filter, which uniquely identified the circular region of interest. The three points were selected to cover the entire filtration area (diameter $34.76 \mathrm{~mm}$ ) with an additional band around the perimeter (see Fig. 5). The circular region was fully covered with overlapping tiles organized in a grid. The dimensions of the tiles were $90 \%$ of the image field size producing a $5 \%$ overlap among adjacent images. The overlaps ensured that no particles were missed but required subsequent processing to avoid double counting of particles in the overlapping margins. In addition, the overlap 
facilitated handling particles which fall close to the edge of an image tile. At the lowest magnification (a nominal $2.0 \mathrm{~mm}$ field-of-view), an image was collected sequentially at each of approximately 500 tiles (see Fig. 3). At increased magnifications of $(1.0 \mathrm{~mm}, 0.5 \mathrm{~mm}$, and $0.25 \mathrm{~mm}$ fields-of-view), images were collected from a randomized subset of tiles. In total, approximately 1700 backscatter and 1700 secondary electron images were collected for each filter. The backscattered electron images were the only ones used for the analysis since they provided the least ambiguous representation of the area of each individual particle and the best contrast. The analysis of the twelve filters in this work entailed over 40,000 images and 29,000,000 particles. The entire data acquisition process (tiling, sub-sampling, stage motion and image collection) was scripted in Python (by Nicholas Ritchie) using a NIST-developed microscope control package called SEMantics [7], an extension to NIST DTSA-II [8]. The automation ensured a high level of consistency among sample sets. In addition, the probe current, the electron beam landing energy, the working distance, and the brightness and contrast settings were held constant for the acquisition of all images

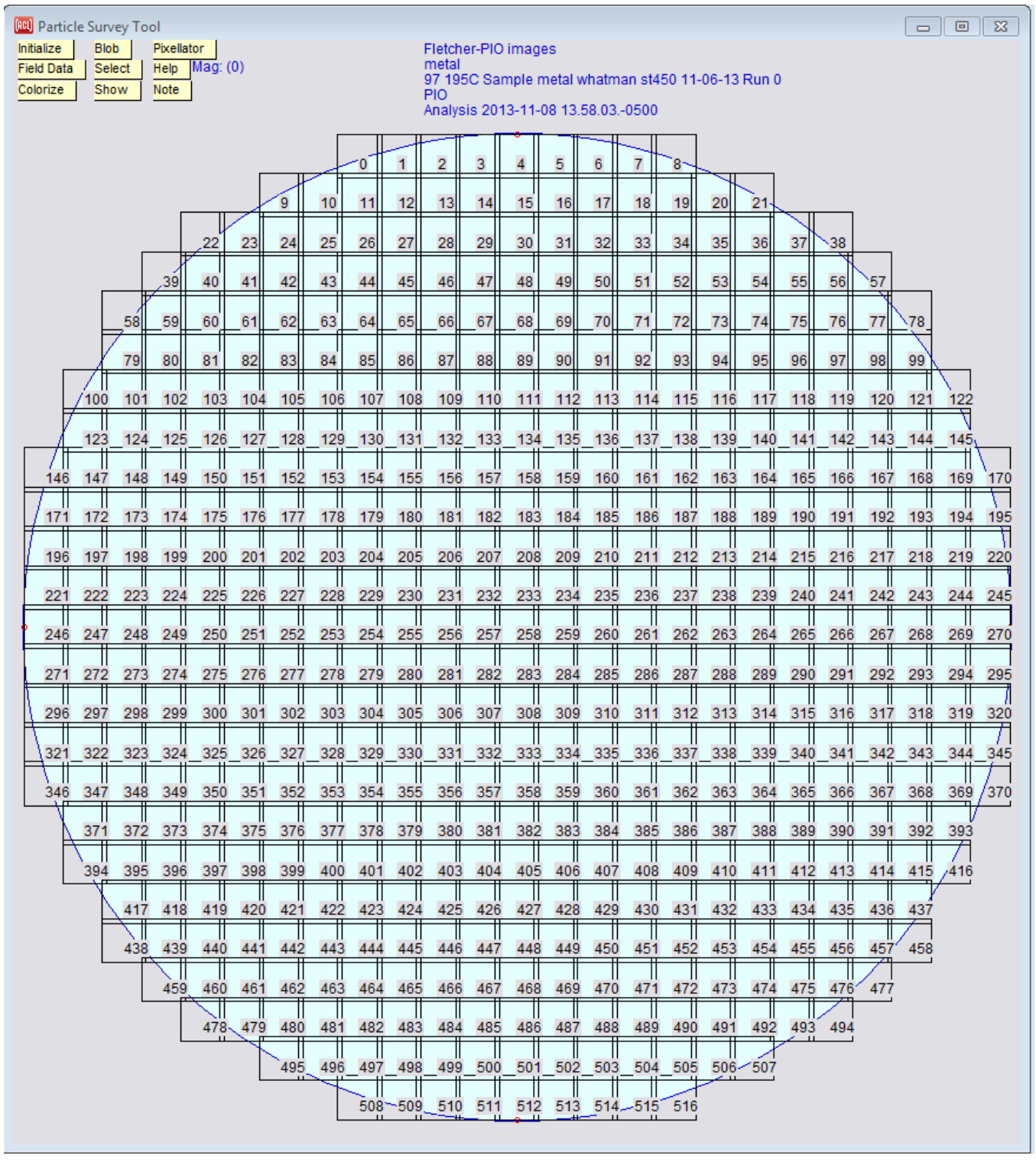

Fig. 3. Set of 517 images collected on a filter at the lowest $(2.0 \mathrm{~mm})$ magnification. Double edges indicate field overlap. 
For interior images the $5 \%$ overlap at each edge causes about $19 \%$ of the area of each image to be duplicated in neighboring images. This might cause approximately $19 \%$ of the particles to be counted more than once. A simple method eliminated double counting using the location of the particle centroids with respect to the mid-line of the overlapping region. We image all the particles on the filter and do not assume that the particles are deposited uniformly. This allows our verification of the data quality among the filter samples to be more robust. The highest confidence is in the $2.0 \mathrm{~mm}$ field-of-view magnification data because the full area was imaged. In contrast, we only imaged a randomly selected subset of fields (see Fig. 4) at $1.0 \mathrm{~mm}, 0.5 \mathrm{~mm}$ and $0.25 \mathrm{~mm}$ and assumed that those fields were representative.

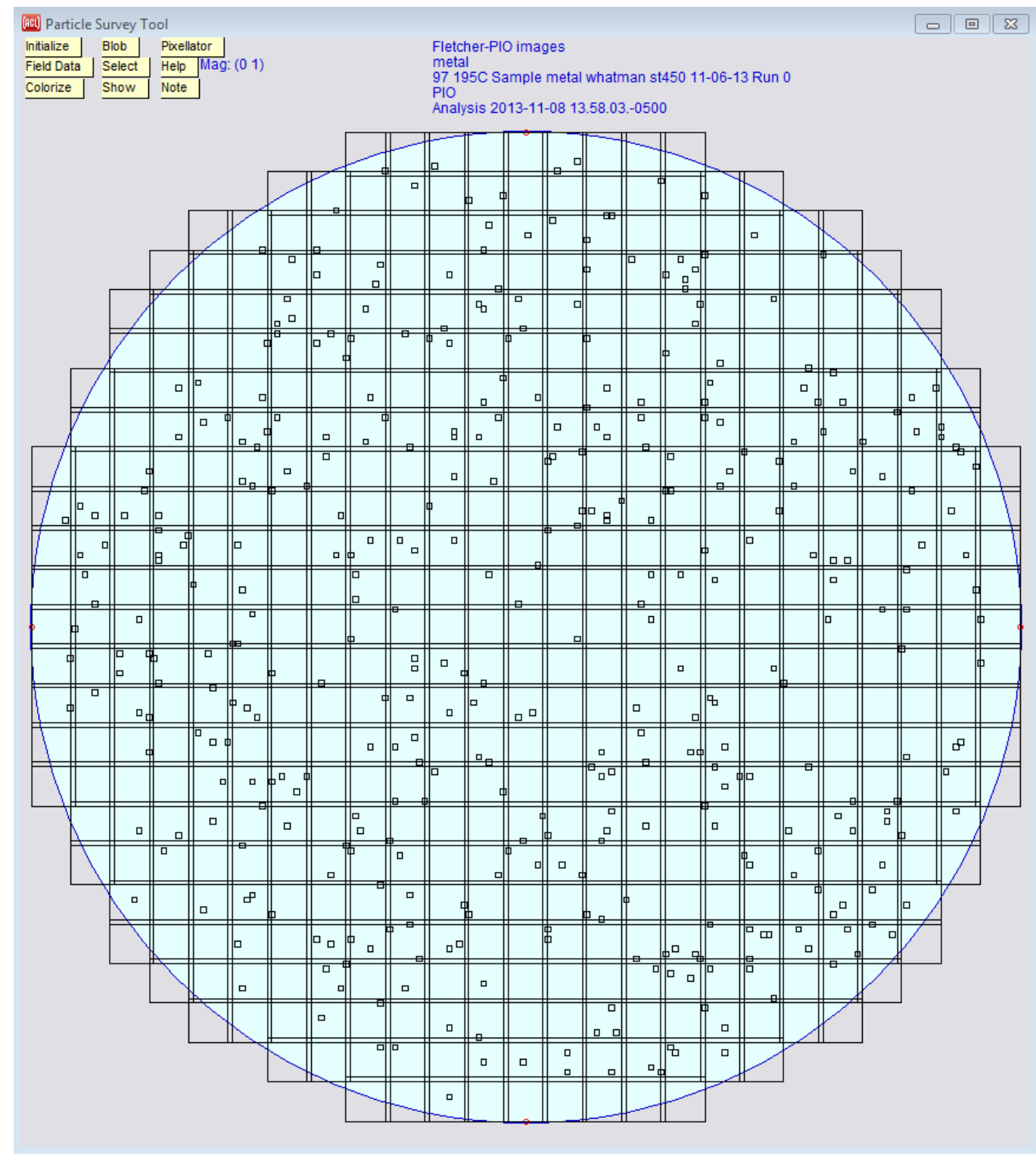

Fig. 4. Filter diagram showing $2.0 \mathrm{~mm}$ fields (large squares) and within them, the randomly selected (with blocked coverage constraints) $0.25 \mathrm{~mm}$ fields (small squares) over the entire filter. 


\section{Results and Discussion}

\subsection{Process Verification}

Characterization using optical particle counting indicated that there was a high degree of bottle-to-bottle homogeneity. It naturally followed that our SEM-based data should have high precision if our measurement process were representative and reproducible. Fortunately, we were able to use one step of the measurement process to validate our results. For all the filtered samples, we imaged the entire filter surface at the low magnification ( $2.0 \mathrm{~mm}$ field-of-view) and consequently always counted and sized every filtered particle above certain image gray level threshold and particle size in the amount of fluid filtered. We determined the mass of the hydraulic fluid filtered. We constructed size distributions from a number of low magnification image data sets, normalized the distributions to the mass of hydraulic fluid (particles /gram of hydraulic fluid), and compared the resultant size distributions over a number of bottles of SRM 2806b. This gave us an internal way of comparing filter-to-filter and bottle-to-bottle variation using a part of the necessary collected data. Ideally, these size distributions should be the same because they were normalized to their respective sample mass. We found that comparisons of a number of size distributions from different filters indicated that we had high variation in the overall measurement process.

We systematically dissected the process to find the cause of the variation. First, we tested the microscopy using experimental design to find what SEM parameters would introduce the most variation on the results. The SEM proved not to be the cause of the variation, so we concentrated on the filter preparation. We noticed that the polycarbonate filters were electrically charged presumably because we filtered a dielectric fluid (hydraulic fluid) and washed the filtrate with a dielectric solvent, heptane. We were concerned because the filter was difficult to manipulate, providing opportunity for particle loss from the filter surface. To reduce the static charge, we increased the electrical conductivity of the solvent by adding a hydraulic fluid additive and used a metal filter holder. Addition of the antistatic agent to both the hydraulic fluid and the heptane greatly reduced this charging. Another aspect of electrical charge that concerned us was the possibility of developing concentrated charge domains on the glass filtration apparatus. We could envision particle loss to the glass due to these highly charged regions. By replacing the glass holder with a metal filter holder and grounding the metal we minimized the particle loss due to charge.

The last step in the filter preparation was to apply a gold coating using an argon plasma coater. Originally we mounted the filter, which was only $20 \mu \mathrm{m}$ thick, in the normal recommended manner, $4 \mathrm{~cm}$ away from the Ar plasma, a zone of high electric fields. To reduce the chances of losing charged particles in the high electrical field, we mounted the filter much farther away $(13 \mathrm{~cm})$ from the plasma zone. We also introduced the argon leak at the start of the pump down cycle, to eliminate the harsh valve snap and gas rush which might also have been removing some particles.

\subsection{Image Analysis}

There are several aspects of the image analysis that are critical to the success of the certification process for SRM 2806b: making a montage image of the entire filter, delineation of the filtration area, particle image analysis and Geller standard analysis. The image analysis was accomplished using the Lispix [9] code which is freely-available software developed by one of the authors (D. S. Bright) with custom utility developed enhancements for this project. After an image set was acquired, a filter map of the sample surface was made, with an example shown in Fig. 5. The software made a mosaic of all the $2.0 \mathrm{~mm}$ fieldof-view images to give an accurate representation of the filter surface. This was invaluable because it allowed the inspection of the images and the filter before proceeding with detailed analysis. The filtration process was faulty many times, and this inspection step showed when there was a problem. We excluded the results of samples of faulty filtration. Inhomogeneous particle coverage was observed (see Fig. 5), possibly due to changes in fluid flow or sample washing. Moderate inhomogeneity was not a problem because we covered the entire filtration area at low magnification and counted all of the particles. We scaled the high magnification $(1 \mathrm{~mm}, 0.5 \mathrm{~mm}$, and $0.25 \mathrm{~mm}$ ) field-of-view data sets to the $2.0 \mathrm{~mm}$ data set using the filter area represented by each set. 


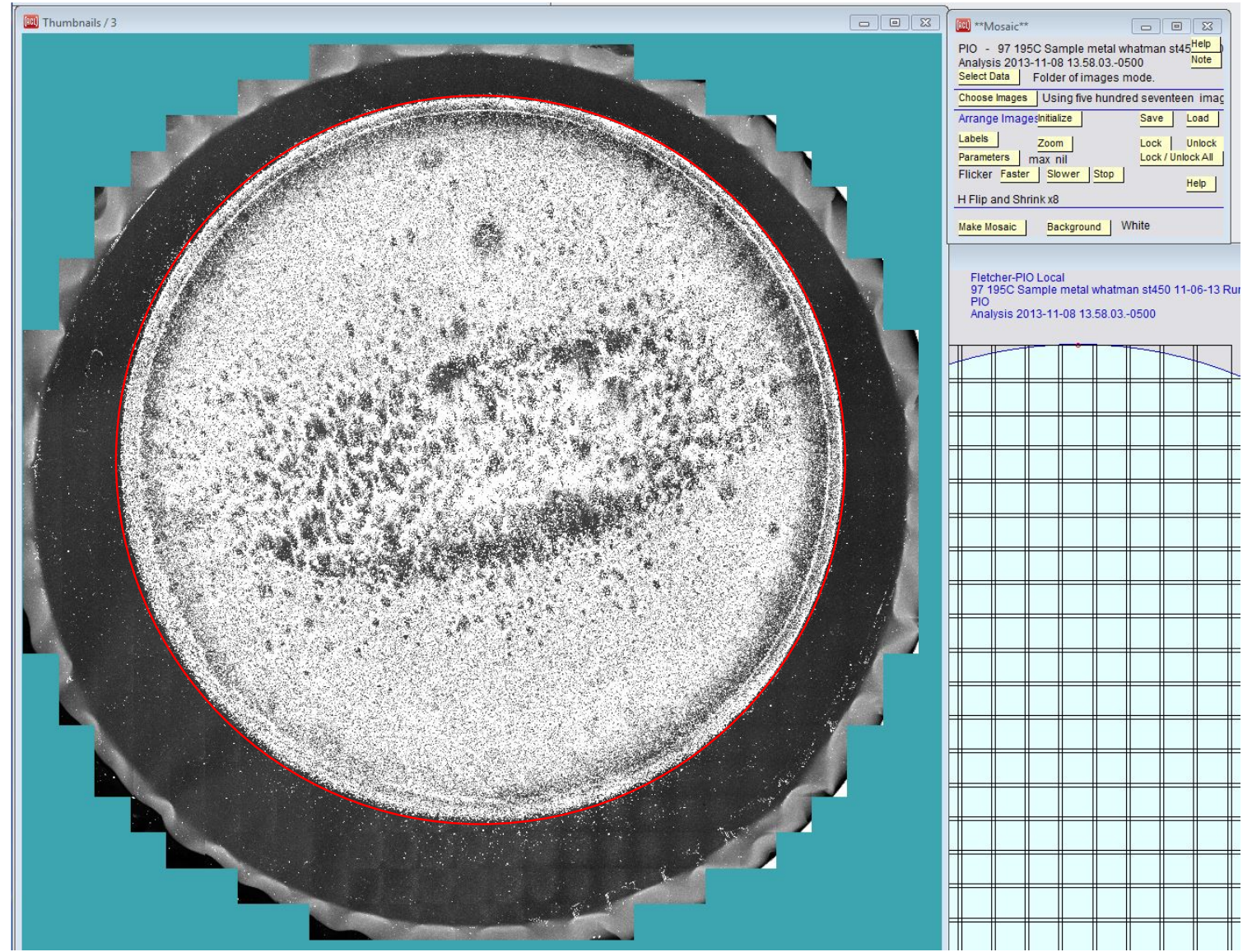

Fig. 5. Tiled SEM image of the entire filter. Filtration area is inside the manually drawn red circle.

As mentioned above, all of the low magnification SEM images overlapped. Image overlap was used to ensure that we imaged and counted all the particles on the filter and thus all the particles in a known volume of hydraulic fluid. To measure the width of the overlap, i.e., the borders of the images that show the same area, we moved two central and adjacent images slightly by hand with respect to each other until the same particles in the margins matched. Matching all other image pairs involved cross correlating central square segments of the borders, using initial offsets from adjacent and previously matched pairs. This approach allowed us to measure the overlaps to within a pixel in the image field. The microscope stage coordinates gave image overlaps to within five or so pixels, which we refined to within a pixel using cross correlation measurements of the overlapping image borders. The left border of one image is shown diagrammatically in Fig. 6, with the original image boundary in red, the trimmed-for-overlap boundary in blue, and the other image boundaries in gray. The blue boundary simply bisects overlapping margins. The software assigns a particle within the overlap region to the image in which it is most central, as shown in Fig. 7. When a particle is in the overlap region and thus appears in both images, the image in which the centroid of the particle is inside the blue trim line is the image for which the particle is counted. Of course, there really are four trim lines, one for each edge, and the centroid must be inside the rectangle that the four trim lines form. If the particles are smaller than the overlap region, then there are no edge effects in that all of the particles are entirely within at least one image. 


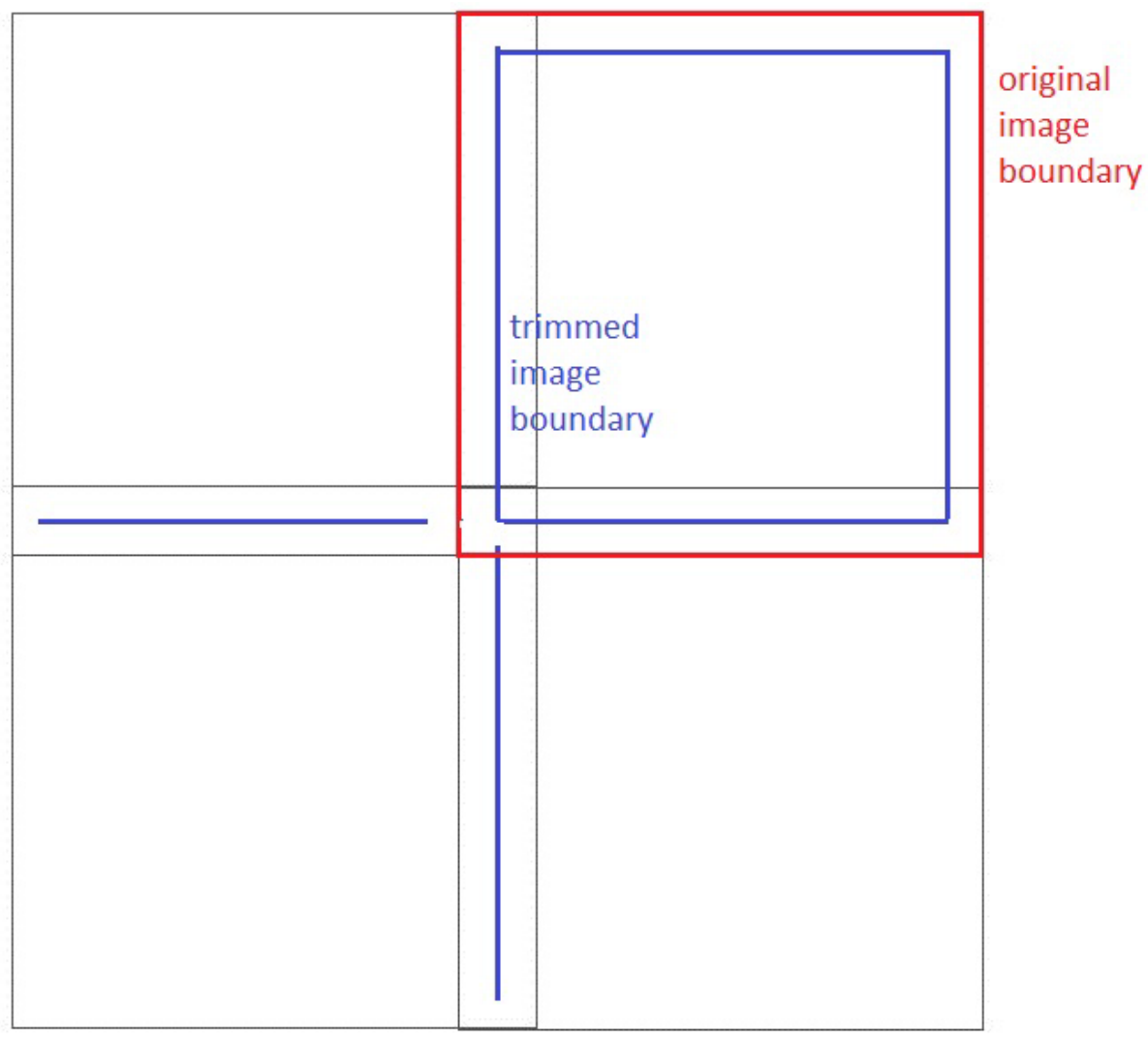

Fig. 6. Diagram showing image overlap.

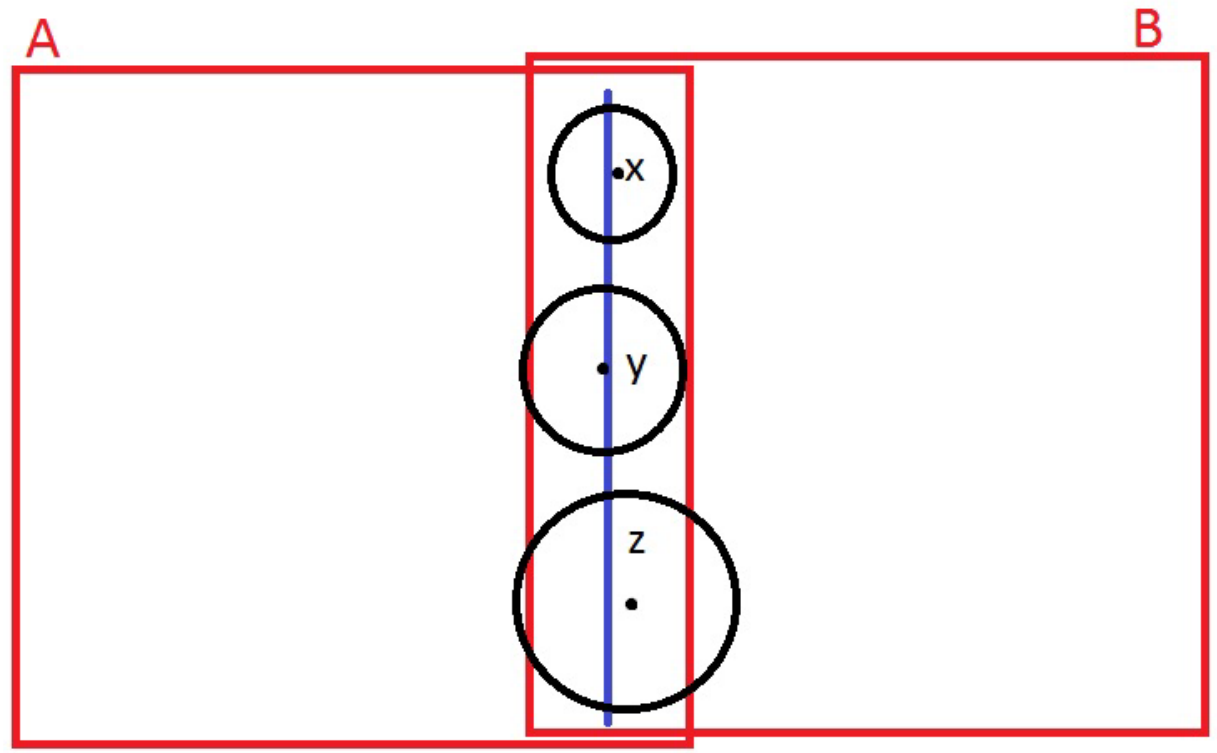

Fig. 7. Diagram that illustrates assignment of particles in the overlap region to image A or image B. Particles are shown as circles with centroids represented as black dots. 
For example, in Fig. 7, the centroid of particle $x$ is to the right of the trim line, and is counted in image B. The centroid of particle $y$ is to the left of the trim line, and is counted in image A. Note that it overlaps the boundary of image B, and would not be counted in image B in any case. Particle $z$ is not completely within either image because it is large enough to span the overlap region. The overlap region is nominally 100 pixels or $200 \mu \mathrm{m}$ wide and to have a $z$ particle condition, the particle would have to be quite large ( $>30 \mu \mathrm{m}$ diameter). There are not many of these large particles, so large particles (particle $\mathrm{z}$ ) boundaries are improbable.

\subsection{Particle Segmentation}

The backscattered electron images show the silicate particles as light rounded objects with sharp edges against the darker background of the polycarbonate filter. Image intensity is affected by both mean atomic number and sample thickness, so small or thin particles have lower contrast approaching the background texture of the filter.

Segmentation involves setting an intensity threshold [10] where all pixels above the threshold are considered to be particle pixels, i.e., part of a particle, and all those below are considered to be background pixels, i.e., not part of a particle but of the texture of the filter. Our algorithm finds the lowest threshold level that avoids selecting too much background, while still selecting as many of the small low intensity particles as possible. The texture of the background happens to be fine grained, that is, there are no features as large as even the smallest particles of interest. This works to our advantage in that low thresholds select both dim particle pixels and dim background pixels, but these are separated and the background pixels are automatically rejected when segmenting. For particles to be counted, they must have at least from three to ten contiguous particle pixels, depending on the magnification.

Consider the image in Figs. 8a-e, with pixel intensities ranging from 0 to 255, and threshold decreasing from Fig. 8a to Fig. 8e. As the threshold is lowered from the maximum 255 (very few pixels selected), additional pixels are selected, which are all particle pixels because the brightest pixels are always in particles. In Fig. 8a, most particles are selected, but some clearly are not selected, appearing in the figure either as small white blobs or as tiny white holes in already selected particles. By visual inspection and by the nature of the sample, we know that the particles do not have small holes. At this threshold, the area or pixel count of most particles is artificially low. Lowering the threshold further (Fig. 8b) selects almost all of the particles and very little background. Close inspection (not shown) suggests, though, that pixels on the edges of the particles, which are the most dim particle pixels, are not yet selected. As the threshold is lowered further (Fig. 8c), selected particles tend not to grow appreciably in area (number of pixels) any more, while the number of background pixels increases. The threshold of Fig. 8c as well as thresholds nearby are all equally useful because particle pixels are now all selected but with very few background pixels. Most of the background pixels that are selected, are isolated (they have no neighboring selected pixels). These background pixels are rejected because they are not part of a contiguous group of three or ten pixels (depending on the magnification) as is the case with particle pixels. As the threshold is decreased even further, the background pixels increase in number and join together (Figs. 8d and 8e), mimicking the real particle pixels and result in false positives and in coalesced particles.

To show this characteristic of the images mathematically, we present a plot of particle area fraction vs. threshold (Fig. 9). The Particle Area Fraction (PAF) is the number of selected particle pixels (part of a contiguous group of pixels) normalized by the number of all selected pixels. Figure 9 shows the PAF, $\mathrm{P} / \mathrm{N}$, plotted vs. threshold, where $\mathrm{N}=$ total selected pixels, i.e., number of pixels above threshold, and $\mathrm{P}=$ 'particle' pixels above threshold. $\mathrm{P}=\mathrm{N}-\mathrm{S}$, where $\mathrm{S}=$ single or isolated selected pixels. So, for any threshold, $\mathrm{P}$ represents blobs or clusters of pixels, which may or may not represent real particles, and $\mathrm{S}$ represents single pixels, which may or may not represent background. In the region around point c, P does correspond to real particles and S to background. Points a-e in Fig. 9 corresponds to Figs. 8a-e, respectively.

Following the curve in Fig. 9 from right (threshold is 255) to left (threshold is zero), as the threshold level decreases, selected (red) pixels are all particle pixels, around $80 \%$ being connected one to another and the rest isolated $(\mathrm{PAF} \approx 0.8)$. These are all particle pixels because real background pixels are well below these intensities (points $a$ to $b$ ). At thresholds lower than point b, few additional particle pixels are 


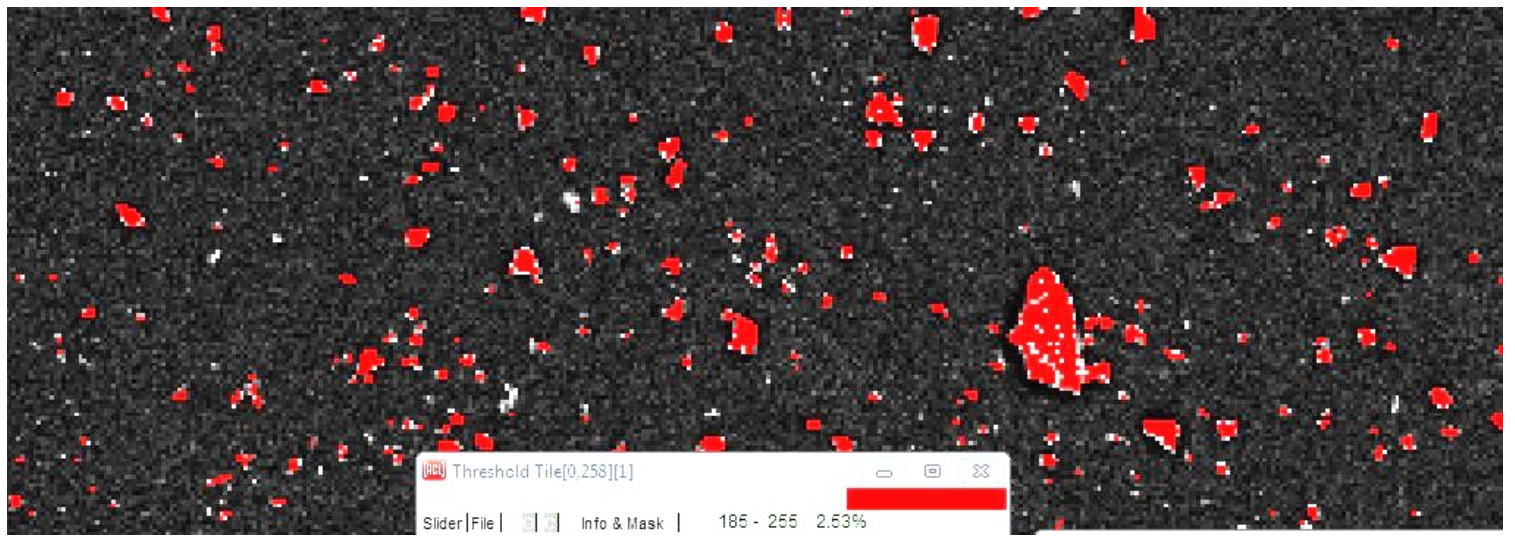

Fig. 8a. Threshold for point 'a' in Fig. 9, selected pixels are shown in red. Threshold is too high: because some low intensity (dim) particles are not selected (red) and most particles are not completely selected (white holes or edges).

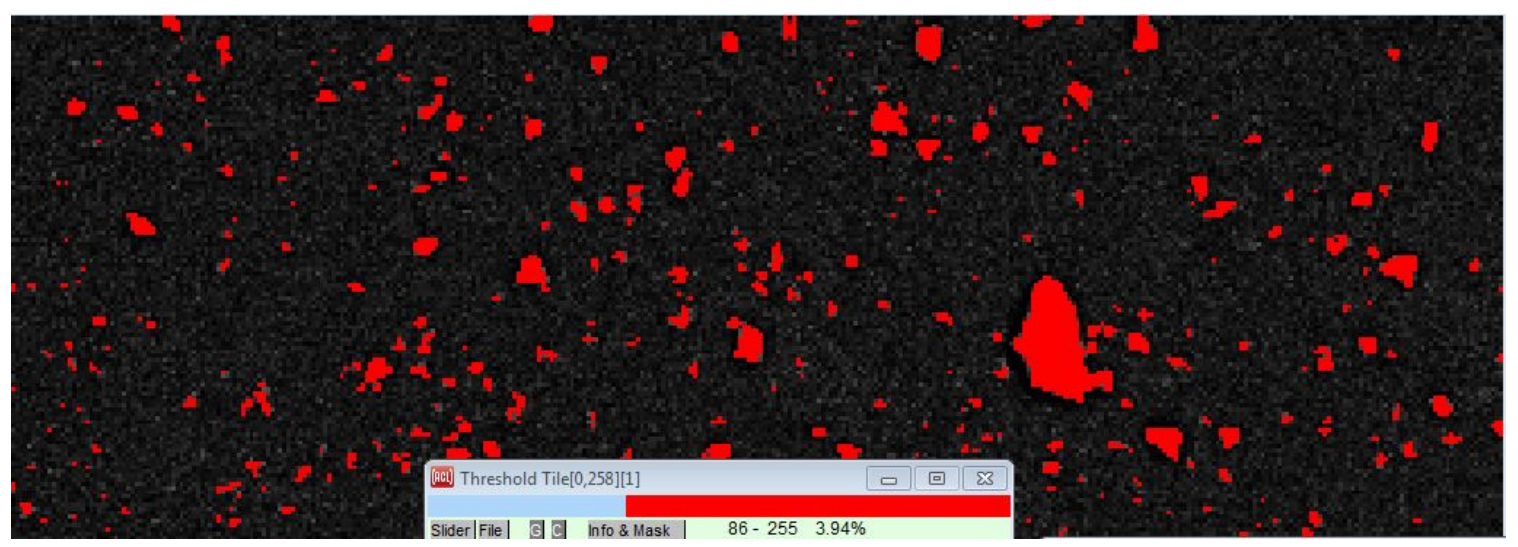

Fig. 8b. Threshold for point ' $b$ ' in Fig. 9. Most particles completely selected.

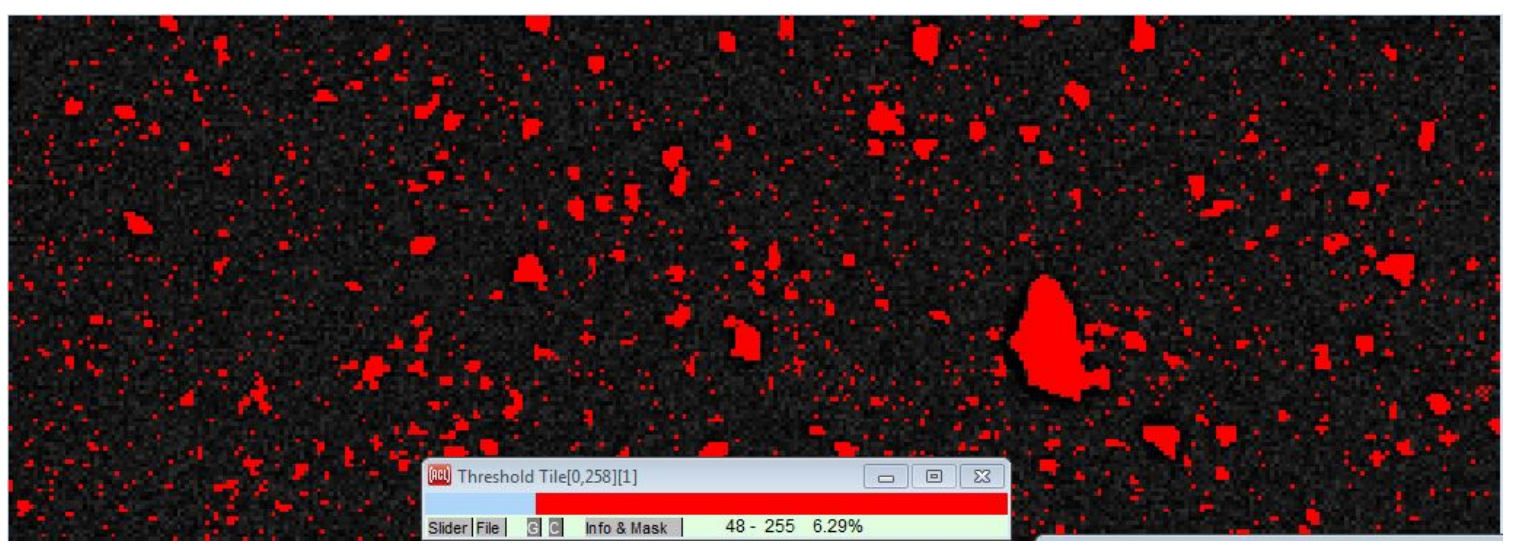

Fig. 8c. Threshold for point ' $c$ ' in Fig. 9. Optimal point for objects and background. Even dimmest particles selected, but background features are not. 


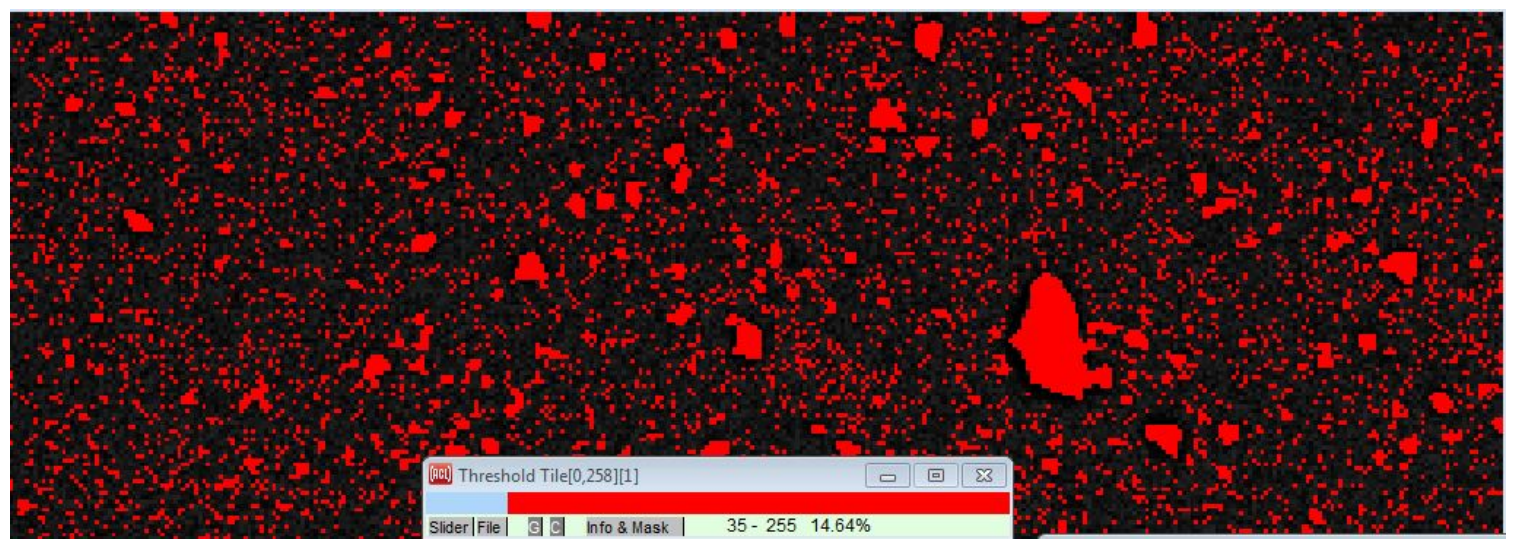

Fig. 8d. Threshold for point ' $d$ ' in Fig. 9. Threshold too low: too many background pixels selected, which are starting to form clusters that mimic particles.

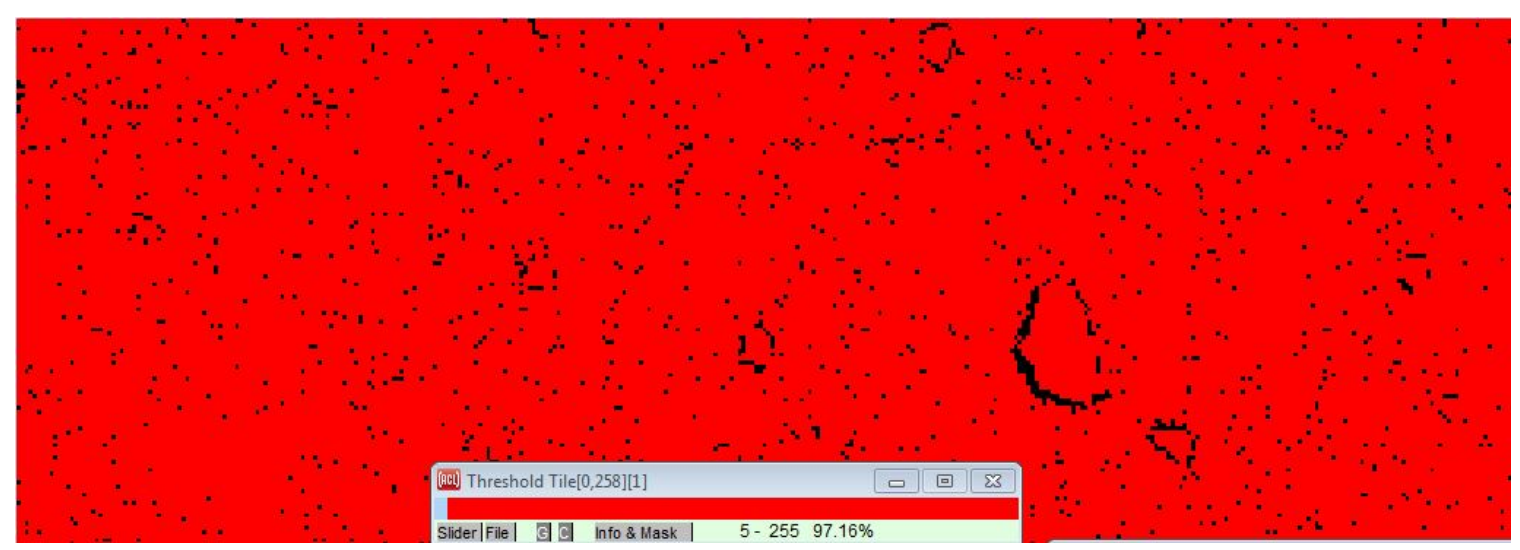

Fig. 8e. Threshold for point ' $e$ ' in Fig. 9. Threshold far too low: particles and background all fused together.

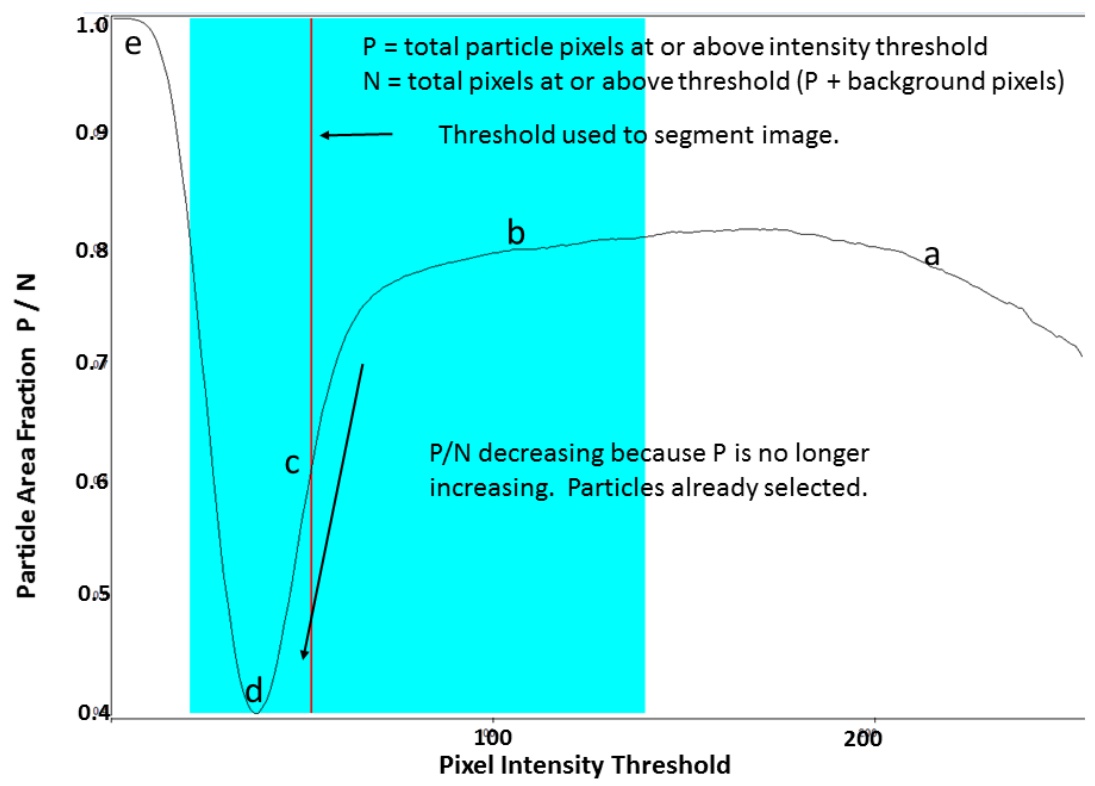

Fig. 9. Plot of particle area fraction $\mathrm{P} / \mathrm{N}$ vs. pixels intensity threshold. Corresponding image samples above in Figs. 8a-e. Appropriate threshold is at point $c$. 
being selected along with an increasing number isolated pixels, which causes the PAF to drop. This continues through point $\mathrm{c}$, the threshold, to point $\mathrm{d}$, where the newest selected pixels are background, and are isolated: $\mathrm{N}$ increases through an increase in S, P is static, thus the PAF decreases. Between points $\mathrm{d}$ and e, the newly selected (background) pixels increase rapidly, and coalesce. The resulting blobs mimic particles - the pixels form connected groups although these groups now do not represent particles but fluctuations in the background signal from the filter. Finally, at point e, the threshold equals zero, and $\mathrm{P}=\mathrm{N}$ and the $\mathrm{PAF}=1.0$.

The threshold used to segment each image is a point on the PAF for that image that is half way up from the minimum at point $d$ to the maximum between points $b$ and $a$. This is the right edge of the cyan rectangle, which is the largest region enclosing the global minimum without including other smaller local minima to the right of point $b$, where the PAF is noisy because few pixels are selected there.

\section{Image Analysis on the Geller Standard to Calibrate Pixel Size}

SEM images of the Geller scale setting standard were taken with each filter sample, at each magnification used in the imaging and analysis. We calibrated the scale by measuring the length in pixels for the two most widely separated traces on the scale setting standard in the horizontal and vertical directions, and then associating these distances in pixels with the known real distances or lengths from NIST interferometer measurements. Figure 10 shows the area of the standard that we used for calibration of horizontal distances. Since only the red lettered features were calibrated by the NIST interferometer, we extended the NIST calibration to the blue lettered features by measuring their positions in a large image of the scale setting standard (8 times normal resolution).

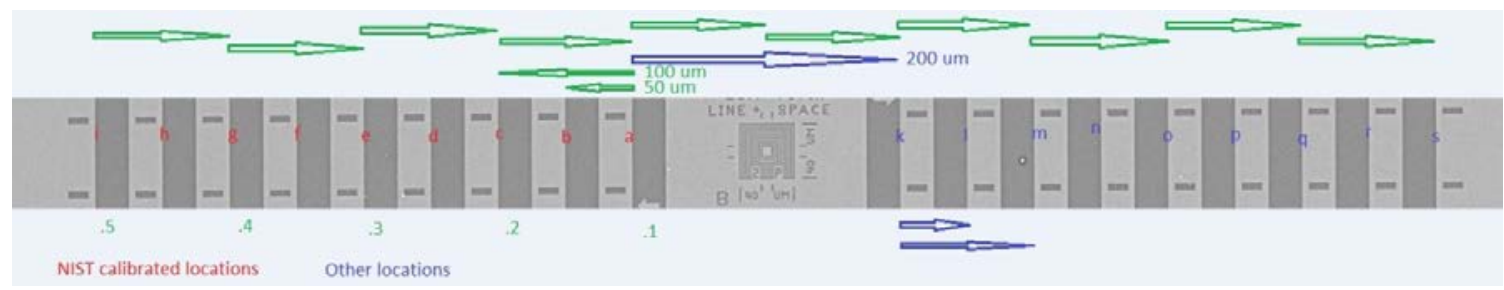

Fig. 10. Horizontal NIST calibrated area of standard: left half of the image (between horizontal dashed lines—see Fig. 2). Measurements on the left extended to the right half of image. Green—nominal $100 \mu \mathrm{m}$ distances. Red letters: NIST-traceable LSI calibrated features, distances measured—from feature a to features $b$ through $i$. Blue letters: secondary features.

\section{Calibration of Pixel Size}

We calculated feature dimensions in micrometers in the particle images by comparing their dimensions in pixels with those of features in the scale standard whose real lengths are known. This involved measuring the left or right edges of the dark lines (as appropriate, see Fig. 10) by hand to determine the actual distance that a single pixel represents. To do this, we zoomed images of the lines so that the region between the upper and lower dashed lines (Fig. 10) were about the height of the computer screen, and then clicked on the edge of the trace 32 times at arbitrary locations along the trace. Table 2 shows the horizontal measurements for one of the filters (9 edge clicks shown), along with the averages and sample standard deviations. Note that the features used for the calibration reflect the most widely spaced ones for that magnification.

Calibrated pixel length values for all the filters in $\mu \mathrm{m} /$ pixel for the $2.0 \mathrm{~mm}$ field-of-view magnification are in Table 3. True pixel area was calculated using the product of the horizontal and vertical values. 
Table 2. Pixel calibration measurements for one filter, five magnifications (only four actually used). Table of actual edge positions, position averages, lengths between features, and pixel sizes in $\mu \mathrm{m}$ from the length standard. Likewise, the vertical features of the scale setting standard provided a conversion to metric for the pixel height. It was found that the horizontal distance was slightly larger than the vertical due to a very slight offset in the SEM scan isotropy calibration. Letters denote positions along the scale setting standard shown in Fig. 10.

\begin{tabular}{|c|c|c|c|c|c|c|c|c|c|c|}
\hline \multirow{3}{*}{$\begin{array}{l}\text { Mag } \\
\text { Position }\end{array}$} & \multicolumn{10}{|c|}{ Horizontal Positions, Pixels } \\
\hline & \multicolumn{2}{|c|}{$250 \mu \mathrm{m}$} & \multicolumn{2}{|c|}{$500 \mu \mathrm{m}$} & \multicolumn{2}{|c|}{$1000 \mu \mathrm{m}$} & \multicolumn{2}{|c|}{$1200 \mu \mathrm{m}$} & \multicolumn{2}{|c|}{$2000 \mu \mathrm{m}$} \\
\hline & $\mathrm{k}$ & l & c & $\mathrm{n}$ & g & $\mathrm{s}$ & $\mathrm{i}$ & $\mathrm{s}$ & i & $\mathrm{s}$ \\
\hline & 792 & 999 & 35 & 962 & 67 & 994 & 55 & 913 & 238 & 752 \\
\hline & 792 & 999 & 36 & 962 & 67 & 994 & 56 & 913 & 238 & 752 \\
\hline & 793 & 999 & 36 & 961 & 68 & 994 & 55 & 913 & 239 & 752 \\
\hline & 793 & 998 & 35 & 961 & 68 & 993 & 55 & 913 & 239 & 752 \\
\hline & 793 & 1000 & 35 & 961 & 68 & 993 & 56 & 913 & 239 & 752 \\
\hline & 792 & 999 & 36 & 962 & 67 & 993 & 55 & 913 & 239 & 752 \\
\hline & 792 & 999 & 36 & 962 & 67 & 993 & 55 & 913 & 239 & 752 \\
\hline & 793 & 998 & 35 & 962 & 67 & 994 & 55 & 913 & 239 & 752 \\
\hline & 793 & 997 & 35 & 962 & 68 & 994 & 55 & 913 & 238 & 752 \\
\hline & 793 & 998 & 35 & 962 & 67 & 994 & 56 & 913 & 238 & 752 \\
\hline Mag & \multicolumn{2}{|c|}{250} & \multicolumn{2}{|c|}{500} & \multicolumn{2}{|c|}{1000} & \multicolumn{2}{|c|}{1200} & \multicolumn{2}{|c|}{2000} \\
\hline Position & $\mathrm{k}$ & l & c & $\mathrm{n}$ & g & $\mathrm{s}$ & $\mathrm{i}$ & $\mathrm{s}$ & $\mathrm{i}$ & $\mathrm{s}$ \\
\hline average & 792.4 & 998.4 & 35.4 & 961.4 & 67.5 & 993.6 & 55.5 & 913 & 238.3 & 752.0 \\
\hline \multirow[t]{2}{*}{ s.d. } & 0.5 & 0.7 & 0.5 & 0.5 & 0.5 & 0.5 & 0.5 & 0 & 0.5 & 0.2 \\
\hline & $\mathrm{k}-\mathrm{l}$ & & $c-n$ & & g-s & & i-s & & i-s & \\
\hline len - pixel & 205.9 & & 926.0 & & 926.1 & & 857.5 & & 513.7 & \\
\hline s.d. - px & 1.5 & & 1.3 & & 1.3 & & 0.9 & & 0.9 & \\
\hline rsd & 0.0071 & & 0.0013 & & 0.0013 & & 0.0015 & & 0.0018 & \\
\hline$\mu \mathrm{m} /$ pixel & 0.2433 & & 0.4852 & & 0.9704 & & 1.1647 & & 1.9444 & \\
\hline s.d. & 0.0019 & & 0.0008 & & 0.0013 & & 0.0013 & & 0.0035 & \\
\hline rsd & 0.0080 & & 0.0016 & & 0.0014 & & 0.0011 & & 0.0018 & \\
\hline
\end{tabular}

\section{Uncertainty Due to Digital Pixel Representation}

The particles measured by image analysis are real objects with curved outlines represented in the images by square pixels. This representation introduces uncertainty as illustrated in Fig. 11 which shows a disc represented by square pixels. The dark colored pixels are outside the circle, the white pixels are $100 \%$ inside the circle and pixels of varying thermal scale color are partially inside, partially out. Depending upon the threshold level, the area of the disc will be either over estimated or under estimated. The edge pixels are obviously the most important. As the object size increases or the magnification increases, more pixels are used to represent the object, and the uncertainty in the representation decreases. In the thresholding process, a pixel is either counted or not counted (in or out). There are no partial pixels allowed in the area representation once a threshold gray level has been identified. 
Table 3. Metric width and height of pixels for $2.0 \mathrm{~mm}$ field-of-view magnification

\begin{tabular}{ccccc}
\hline & \multicolumn{2}{c}{ Horizontal } & \multicolumn{2}{c}{ Vertical } \\
Filter & $\begin{array}{c}\text { Value } \\
\mu \mathrm{m} / \text { pixel }\end{array}$ & $\begin{array}{c}\text { SD }(\mathrm{n}=32) \\
\mu \mathrm{m} / \text { pixel }\end{array}$ & $\begin{array}{c}\text { Value } \\
\mu \mathrm{m} / \text { pixel }\end{array}$ & $\begin{array}{c}\text { SD (n=32) } \\
\mu \mathrm{m} / \text { pixel }\end{array}$ \\
\hline 71 & 1.960809 & 0.003002 & 1.959739 & 0.002923 \\
74 & 1.959246 & 0.001711 & 1.95890 & 0.002509 \\
75 & 1.959246 & 0.001711 & 1.956507 & 0.003006 \\
76 & 1.968295 & 0.002214 & 1.966234 & 0.002401 \\
77 & 1.968295 & 0.002509 & 1.968045 & 0.002589 \\
78 & 1.971087 & 0.002337 & 1.968045 & 0.003501 \\
79 & 1.965632 & 0.001852 & 1.962981 & 0.002965 \\
80 & 1.966236 & 0.000781 & 1.963101 & 0.001157 \\
94 & 1.959366 & 0.001792 & 1.956149 & 0.001153 \\
95 & 1.962494 & 0.001404 & 1.958780 & 0.002946 \\
96 & 1.994455 & 0.002308 & 1.990668 & 0.002988 \\
97 & 1.944466 & 0.003533 & 1.939922 & 0.004787 \\
\hline
\end{tabular}

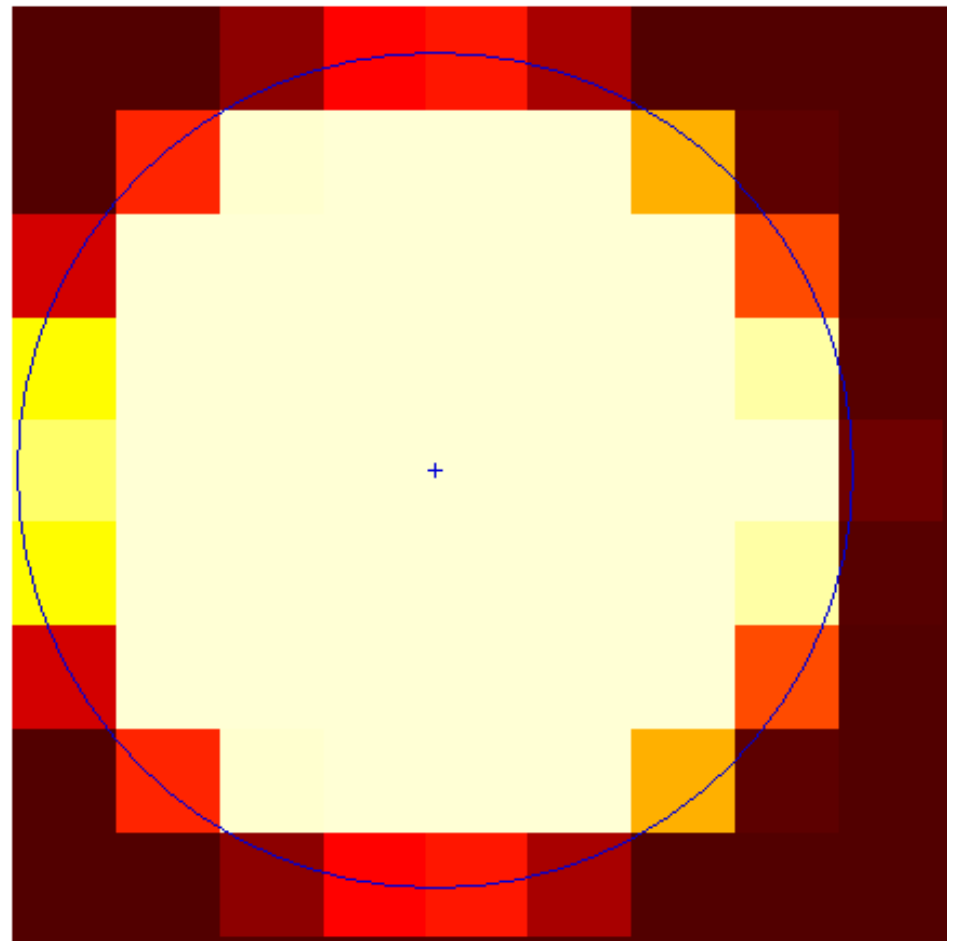

Fig. 11. Disc (outlined in blue) represented by pixels. The thermal color scheme provided shows what degree the pixel is covered by the disc. 
Because of the threshold levels in our analysis, we believe that we overestimate the size of many objects by pixel representation. To verify this hypothesis, we compared the exact same particles imaged at different magnifications in our data set. At the high magnification, the particle is represented with many more pixels than at low magnification so the misrepresentation due to the edge pixels is much less. The hypotheses predict that the particle size determined by the low magnification should be larger than that found at the high magnification. An example is shown in Fig. 12 which gives the percent difference between the particle area $\left(\mu \mathrm{m}^{2}\right)$ found in a particle at the low magnification (2.0 mm field-of-view) when compared to the same particle at the $0.25 \mathrm{~mm}$ field-of-view magnification, plotted against the particle area (in pixels) at low magnification. A fit to equivalent particle circular radius versus error (derived from the area) was done on two independent sets of this data. A coefficient, $c$, was found for the $c /$ radius fit at $99 \%$ confidence limit. The $c /$ radius value and the uncertainty from the random (Type A) processes were fed into the Monte Carlo simulation for the determination of the combined standard uncertainty (discussed later).

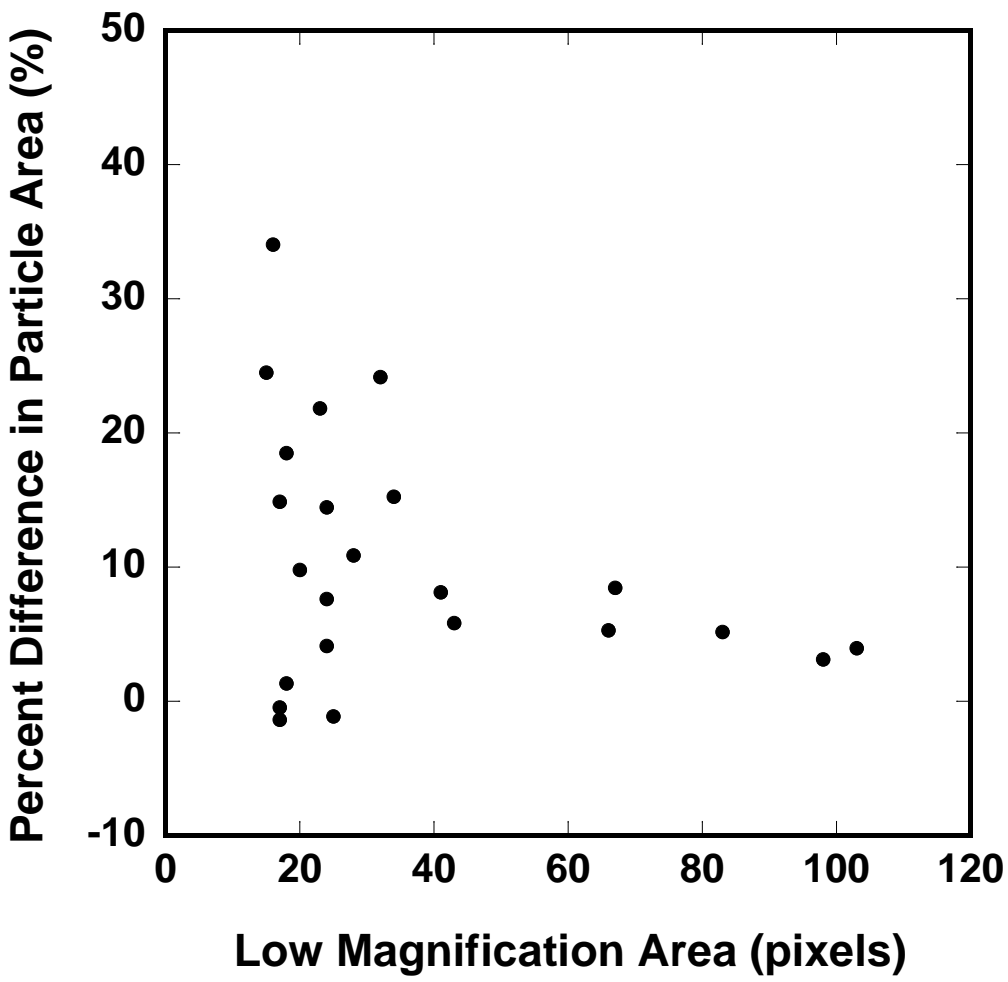

Fig. 12. Percent difference in area in the low magnification image for single particles vs. particle area in pixels for low magnification images.

\section{Certified Values}

For the 12 data sets (filters), we computed the particle concentration (particles/mL) in the hydraulic fluid expressed as a cumulative number size distribution. The particles were binned in the histogram based on their equivalent circular diameter, i.e., diameter of a circular particle having the same projected area as the dust particle. For high-magnification images, a correction was used to account for particle loss at the image edge [11]. No such correction was necessary for the $2.0 \mathrm{~mm}$ field-of-view magnification because of the image overlap strategy. The volume of hydraulic fluid was determined from the mass measurements of the fluid filtered and the density of the fluid. The mean of particle concentration values, as a cumulative number size distribution for all 12 data sets is given in Table 4, column 2 and the standard uncertainty of the mean is in column 3. Column 4 contains the standard uncertainty associated with random sampling, 
column 5 is the standard uncertainty associated with image digitalization, column 6 is the combined standard uncertainty ( $\boldsymbol{u}_{c}$, combined using Monte Carlo simulation), column 7 is the coverage factor, $k$, (back calculated) and the last column is the expanded uncertainty at the $95 \%$ confidence level $\left(U=k u_{c}\right)$.

Table 4. Certified values, sources of uncertainty, and combined standard and expanded uncertainties

\begin{tabular}{|c|c|c|c|c|c|c|c|}
\hline $\begin{array}{c}\text { (1) Projected- } \\
\text { Area } \\
\text { Particle } \\
\text { Diameter }^{\mathrm{a}}\end{array}$ & $\begin{array}{c}\text { (2) Mean } \\
\text { Cumulative } \\
\text { Projected-Area } \\
\text { Particle- } \\
\text { Diameter } \\
\text { Number } \\
\text { Concentration }{ }^{\mathrm{b}} \\
\text { (n=12) }\end{array}$ & $\begin{array}{c}\text { (3) Standard } \\
\text { Uncertainty of } \\
\text { Mean Cumulative } \\
\text { Projected-Area } \\
\text { Particle-Diameter } \\
\text { Number } \\
\text { Concentration } \\
\text { from Sampling } \\
\text { Reproducibility } \\
(\mathrm{n}=12)\end{array}$ & $\begin{array}{l}\text { (4) Standard } \\
\text { Uncertainty in } \\
\text { Projected-Area } \\
\text { Particle Diameter } \\
\text { from Sampling } \\
\text { Reproducibility } \\
\text { (n=12) }\end{array}$ & $\begin{array}{l}\text { (5) Standard } \\
\text { Uncertainty } \\
\text { in Projected- } \\
\text { Area Particle } \\
\text { Diameter } \\
\text { from Image } \\
\text { Digitization }^{\mathrm{d}}\end{array}$ & $\begin{array}{l}\text { (6) Combined } \\
\text { Standard } \\
\text { Uncertainty } \\
\text { for Projected- } \\
\text { Area Particle } \\
\text { Diameter }\end{array}$ & $\begin{array}{c}\text { (7) Coverage } \\
\text { Factor }\end{array}$ & $\begin{array}{l}\text { (8) Expanded } \\
\text { Uncertainty } \\
\text { (U) for } \\
\text { Projected- } \\
\text { Area Particle } \\
\text { Diameter }\end{array}$ \\
\hline$(\mu \mathrm{m})$ & (particles/mL) & (particles/mL) & $(\mu \mathrm{m})$ & $(\mu \mathrm{m})$ & $(\mu \mathrm{m})$ & (1) & $(\mu \mathrm{m})$ \\
\hline 1.0 & 80755 & 1318.7 & 0.0127 & 0.2194 & 0.2198 & 1.1798 & 0.26 \\
\hline 2.0 & 33064 & 530.9 & 0.0229 & 0.2194 & 0.2210 & 1.2476 & 0.28 \\
\hline 3.0 & 17714 & 305.2 & 0.0322 & 0.2193 & 0.2222 & 1.3103 & 0.29 \\
\hline 4.0 & 10864 & 253.5 & 0.0484 & 0.8783 & 0.8799 & 1.1763 & 1.0 \\
\hline 5.0 & 6681.2 & 127.6 & 0.0410 & 0.8788 & 0.8798 & 1.1641 & 1.0 \\
\hline 6.0 & 4210.2 & 82.78 & 0.0467 & 0.8781 & 0.8795 & 1.1749 & 1.0 \\
\hline 7.0 & 2852.3 & 61.18 & 0.0579 & 0.8777 & 0.8802 & 1.1935 & 1.1 \\
\hline 8.0 & 2007.0 & 38.64 & 0.0590 & 0.8792 & 0.8814 & 1.1937 & 1.1 \\
\hline 9.0 & 1476.4 & 27.90 & 0.0643 & 0.8789 & 0.8819 & 1.2031 & 1.1 \\
\hline 10.0 & 1114.8 & 18.00 & 0.0598 & 0.8786 & 0.8809 & 1.1953 & 1.1 \\
\hline 11.0 & 857.22 & 13.61 & 0.0593 & 1.7566 & 1.7581 & 1.1464 & 2.0 \\
\hline 12.0 & 649.63 & 11.09 & 0.0638 & 1.7585 & 1.7599 & 1.1490 & 2.0 \\
\hline 13.0 & 500.66 & 10.12 & 0.0797 & 1.7587 & 1.7605 & 1.1622 & 2.0 \\
\hline 14.0 & 389.26 & 10.74 & 0.1067 & 1.7585 & 1.7626 & 1.1853 & 2.1 \\
\hline 15.0 & 299.96 & 8.79 & 0.1126 & 1.7582 & 1.7618 & 1.1909 & 2.1 \\
\hline 16.0 & 230.39 & 7.98 & 0.1347 & 1.7594 & 1.7658 & 1.2077 & 2.1 \\
\hline 17.0 & 179.37 & 6.99 & 0.1630 & 1.7610 & 1.7707 & 1.2306 & 2.2 \\
\hline 18.0 & 142.77 & 6.09 & 0.1892 & 1.7582 & 1.7702 & 1.2544 & 2.2 \\
\hline 19.0 & 114.45 & 5.12 & 0.2108 & 1.7586 & 1.7746 & 1.2717 & 2.3 \\
\hline 20.0 & 93.177 & 4.53 & 0.2479 & 1.7587 & 1.7796 & 1.3034 & 2.3 \\
\hline 21.0 & 77.143 & 4.14 & 0.2972 & 1.7569 & 1.7867 & 1.3414 & 2.4 \\
\hline 22.0 & 65.135 & 3.53 & 0.3190 & 1.7596 & 1.7928 & 1.3587 & 2.4 \\
\hline 23.0 & 54.701 & 3.2 & 0.3559 & 1.7582 & 1.7994 & 1.3871 & 2.5 \\
\hline 24.0 & 46.830 & 2.95 & 0.4097 & 1.7593 & 1.8163 & 1.4247 & 2.6 \\
\hline 25.0 & 40.307 & 2.64 & 0.4373 & 1.7582 & 1.8228 & 1.4414 & 2.6 \\
\hline 26.0 & 34.677 & 2.39 & 0.4716 & 1.7602 & 1.8355 & 1.4652 & 2.7 \\
\hline 27.0 & 30.094 & 2.17 & 0.5020 & 1.7587 & 1.8435 & 1.4875 & 2.7 \\
\hline 28.0 & 26.006 & 1.98 & 0.5216 & 1.7587 & 1.8480 & 1.4978 & 2.8 \\
\hline 29.0 & 22.490 & 1.79 & 0.5755 & 1.7579 & 1.8669 & 1.5332 & 2.9 \\
\hline 30.0 & 19.698 & 1.64 & 0.6401 & 1.7583 & 1.8957 & 1.5686 & 3.0 \\
\hline
\end{tabular}

${ }^{\text {a }}$ Stable particle projected area diameter $[12],{ }^{2}$ the standard uncertainty components $(u)$, the combined standard uncertainties $\left(u_{\mathrm{c}}\right)$ and the expanded uncertainties $(U)$ in the projected area diameter (95\% confidence interval).

${ }^{\mathrm{b}}$ Number of particles per milliliter of hydraulic fluid greater than the indicated diameter (number per milliliter). This is a mean value for 12 individual filter analyses.

${ }^{\mathrm{c}}$ Type A uncertainties evaluated by statistical methods $(n=12)$.

${ }^{\mathrm{d}}$ Type B uncertainties evaluated by other means.

\footnotetext{
2 The particle size was determined for the dust particles in their most mechanically stable orientation and thus the particle area approaches on average the largest possible value. Literature values for the ratio of mean projected diameters for random and for stable oriented quartz particles (common to ISO Medium Test Dust) is approximately 0.8.
} 


\section{Uncertainty Components}

The expanded uncertainties listed in Table 4 were calculated according to the ISO/JCGM Guide [13]. These uncertainties correspond to the expanded uncertainties in particle projected area diameters and define a confidence interval of approximately $95 \%$. The combined and expanded uncertainties in Fig. 13 were derived using Monte Carlo simulation with inputs from the digitization and sampling reproducibility uncertainties.

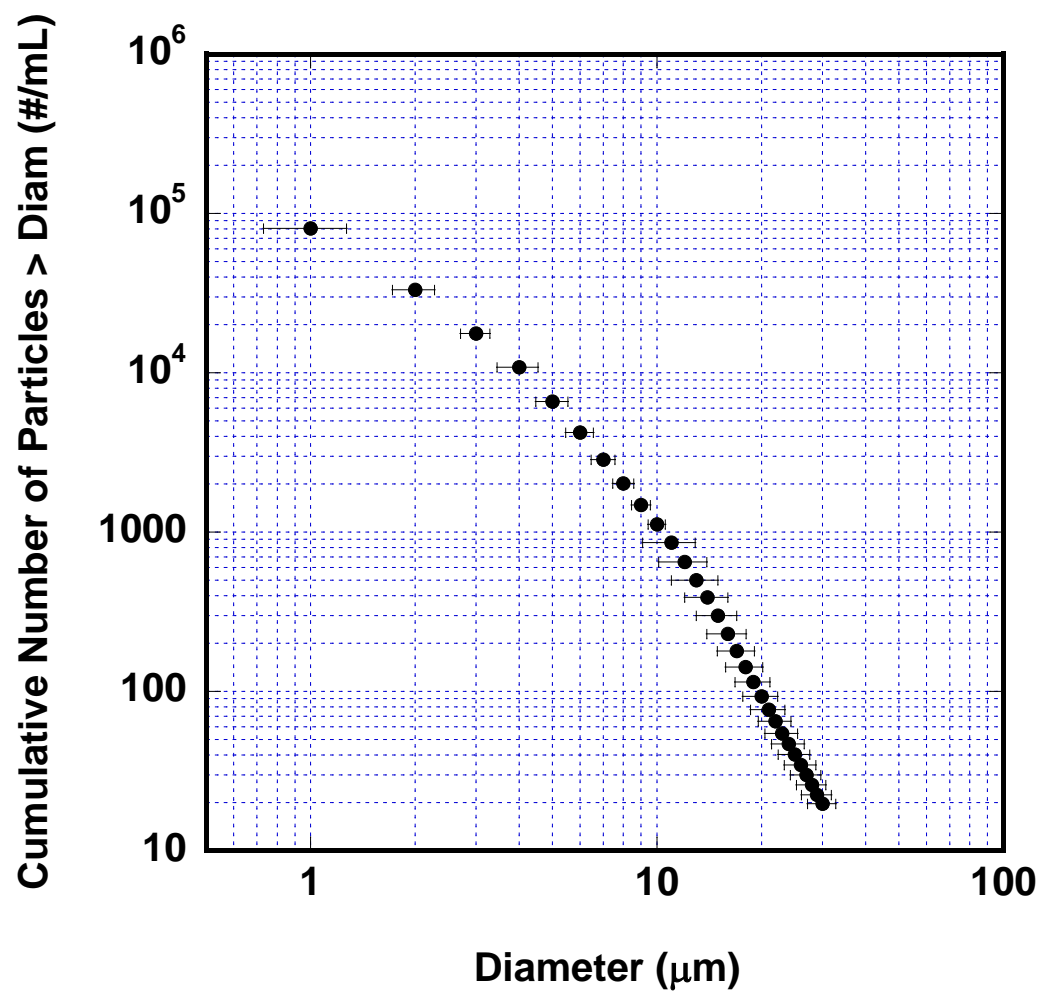

Fig. 13. Plot of cumulative number of particles per milliliter of hydraulic fluid greater than the specified projected area diameter. The expanded uncertainties (U) are shown in the plot. The power law distribution shown in the plot is frequently characteristic of natural fracturing of materials.

The type A (random) and type B (nonrandom or systematic) standard uncertainty components for the measurements arise from a number of sources. Type A uncertainties include (1) particle sampling and counting, (2) hydraulic fluid sampled volume, and (3) SEM magnification variations. Type B uncertainties include (1) digital image representation and processing, (2) Geller scale setting standard, and (3) part of hydraulic fluid volume (density) determination.

There are only 2 main contributors to the measurement uncertainty (shown in Table 5 and Fig. 14), uncertainty due to digital representation (digitization) and random variation in the 12 filters analyzed (sample reproducibility). The uncertainty associated with hydraulic fluid volume has a type A uncertainty component that is included in sampling uncertainty determination. The part that is not random is the estimated bias in the Rudolph Research Density Meter which was determined to be approximately $8 \times 10^{-5} \mathrm{~g} / \mathrm{mL}$ and is too insignificant to impact the uncertainty budget. Length uncertainty impacts the particle size and the area of the field-of-views. The uncertainty in the area of the filter field-of-views is only relevant to the sampling on the filter surface at high magnifications since at low magnification the entire filter area was included. The uncertainty shown in Table 5 for the Geller scale standard correspond to differences in the nominal $50 \mu \mathrm{m}$ to multiple $50 \mu \mathrm{m}$ lengths presented in the scale. The systematic part of 
the length and area uncertainty reported by NIST [4] for the Geller standard was used directly as the traceable length values for the pixel to length $(\mu \mathrm{m})$ conversions for the respective magnifications. The type A uncertainty in the Geller scale measurement (horizontal: $0.0020 \mu \mathrm{m}$ and vertical: $0.0028 \mu \mathrm{m}$, respectively) was included in the random sampling, SEM magnification uncertainty.

Table 5. List of uncertainties, their type either random or systematic and their magnitude

\begin{tabular}{lll}
\hline Source of Uncertainty & Type A (Random) & Type B (Systematic) \\
\hline $\begin{array}{l}\text { Particle sampling, } \\
\text { SEM magnification }\end{array}$ & $0.5 \%$ to $2 \%$ of diameter & \\
Hydraulic fluid density & $1.1 \times 10^{-5} \mathrm{~g} / \mathrm{mL}$ & $8 \times 10^{-5} \mathrm{~g} / \mathrm{mL}$ \\
Digital image analysis & & $6 \%$ to $22 \%$ of diameter \\
Geller scale length & $0.0020 \mu \mathrm{m}$ horizontal & Horizontal: $-0.0018 \mu \mathrm{m}$ to $+0.00158 \mu \mathrm{m}$ \\
& $0.0028 \mu \mathrm{m}$ vertical & Vertical: $-0.0029 \mu \mathrm{m}$ to $+0.0334 \mu \mathrm{m}$ \\
\hline
\end{tabular}

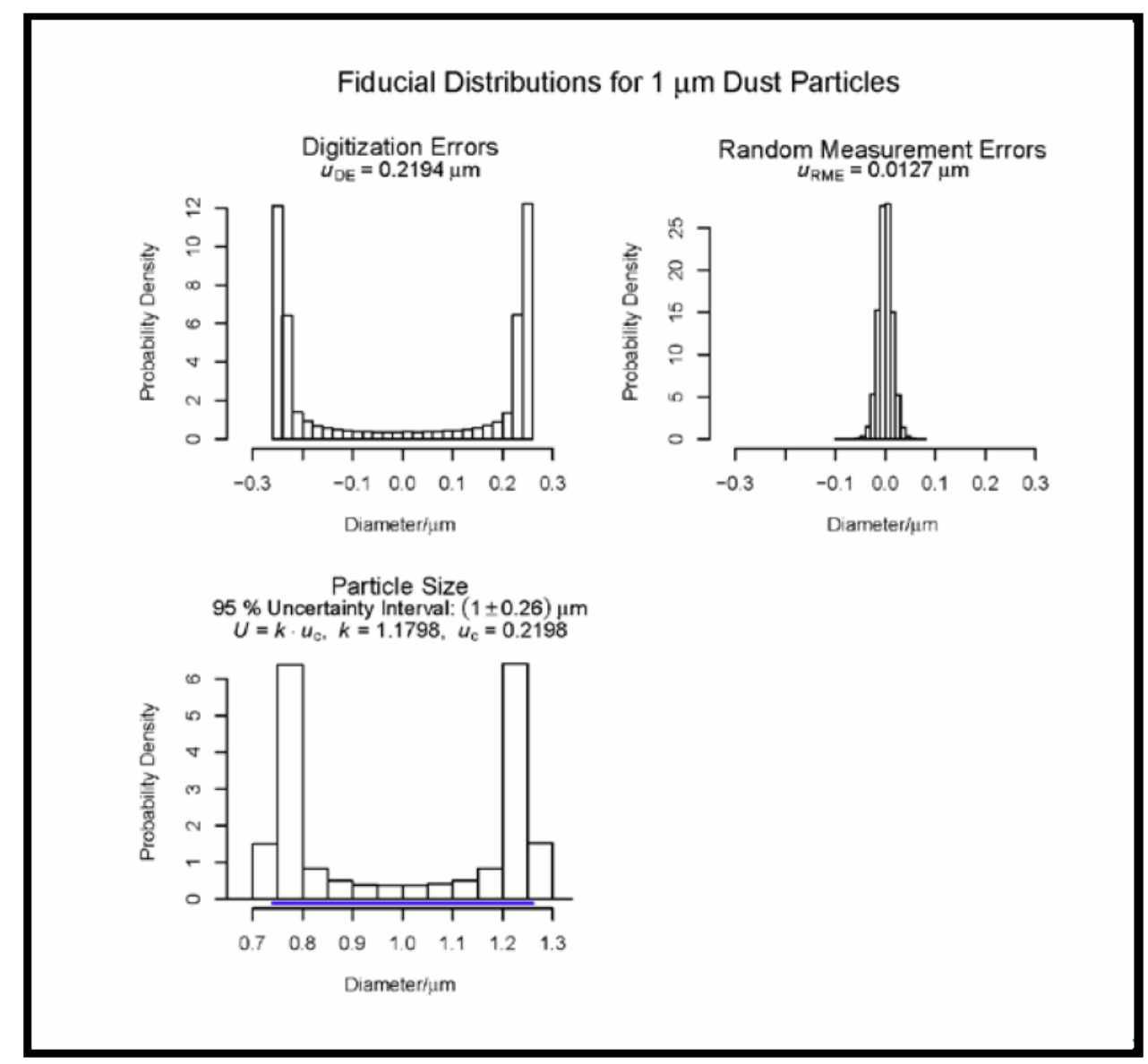

Fig. 14. Monte Carlo simulation used to calculate the combined standard and expanded uncertainties for the main two components, digitization and random sampling. A fiducial distribution is one which is constructed from various considerations so as to reflect the "likely" distribution of errors from a component in a measurement process. For component 1 (digitization error) geometric arguments suggest a U-shaped error distribution as appropriate- the well-known statistical beta distribution was utilized to formally model such U-shapedness. For component 2 (random measurment error), the error distribution was likely symmetric and unimodal, and so the usual Gaussian distribution was assumed. Monte Carlo simulation was then used to form the propagated particle size distribution from which the combined standard and expanded uncertainties were calculated. 


\section{Summary}

SRM 2806b has been a valuable reference material for the Fluid Power Industry and other associated segments of the private sector. NIST collaborated with IFTS in manufacturing SRM 2806b to assure the best possible material would be produced by designing and implementing a series of statistical tests and criteria that had to be met before NIST acceptance. The size of the particles in hydraulic fluid was certified on the basis of their equivalent circular diameter. This was difficult because the particles counted and sized were both irregular in shape (natural mineral particulate material) and polydisperse. We developed methods to guarantee process repeatability, to size and count large number of particles and to estimate the uncertainty in the measurement process. The capability of an automated SEM and advanced image analysis software enabled the certification. Traceability to the meter was provided through the NIST Line Scale Interferometer. A unique feature of the uncertainty analysis with respect to the uncertainty related to the image processing was enabled by the ability to measure pixel area for the exact same particles at multiple SEM magnifications in the particle image set.

\section{Acknowledgments}

The authors would like to acknowledge the valuable contributions of the ad hoc ISO committee that met monthly via teleconference during the development of this Standard Reference Material. The members were Leonard Bensch (US), Garry Bessee (US), Karen Boehme (US), Monica Peterson (US), Nicolas Petilon (France), Christophe Peuchot (France), Terry Phillips (US), Michael Schumacher (Germany), and Barry Verdegan (leader, US). Another contributor we wish to thank is Shlomo Antika (US), an aviation hydraulic fluid expert. We would also like to thank S. Sheckels, PML, NIST Fluid Metrology Laboratory for the careful density determination of the hydraulic fluid and W.B. Penzes for his work to provide NIST traceability for this SRM [4] through the NIST line scan interferometer.

\section{References}

[1] International Organization for Standardization (2010) ISO 11171:2010 - Hydraulic Fluid Power-Calibration of Automatic Particle Counters for Liquids (International Organization for Standardization, Geneva, Switzerland). Available https://www.iso.org/obp/ui/\#iso:std:iso:11171:ed-2:v1:en. Accessed October 24, 2016.

[2] International Organization for Standardization (2016) ISO DIS 12103-1:2016 - Test Dust for Filter Evaluation-Part I: Arizona Test Dust (International Organization for Standardization, Geneva, Switzerland). Available https://www.iso.org/obp/ui/\#iso:std:iso:12103:-1:ed-2:v1:en. Accessed October 24, 2016.

[3] Beers JS, Penzes WB (1999) The NIST length scale interferometer. Journal of Research of the National Institute of Standards and Technology 104(3):225-252. https://doi.org/10.6028/jres.104.017

[4] Penzes WB (2008) Report of Calibration: NIST Test No. 821/276683-08 10X to 50,000X Pitch Standard, Geller MRS-4XY s/n R23-104.

[5] Filliben JJ (1981) DATAPLOT—an interactive high-level language for graphics, non-linear fitting, data analysis, and mathematics. In Proceedings of the 8th annual conference on Computer graphics and interactive techniques (ACM, Dallas, Texas, USA), pp 199-213.

[6] Filliben JJ, Heckert A. DATAPLOT. Available http://www.itl.nist.gov/div898/software/dataplot/. Accessed October 24, 2016.

[7] Ritchie N, Filip V (2011) SEMantics for High Speed Automated Particle Analysis by SEM/EDX. Microscopy and Microanalysis 17(S2):896-897. https://doi.org/10.1017/S1431927611005356

[8] Newbury DE, Ritchie NWM (2015) Performing elemental microanalysis with high accuracy and high precision by scanning electron microscopy/silicon drift detector energy-dispersive X-ray spectrometry (SEM/SDD-EDS). Journal of Materials Science 50(2):493-518. https://doi.org/10.1007/s10853-014-8685-2

[9] Bright DS (1995) MacLispix: A Special Purpose Public Domain Image Analysis Program for the Macintosh. Microbeam Analysis 4:151.

[10] Russ JC (1995) The image processing handbook (CRC Press, Boca Raton, FL) pp 417-446.

[11] Terry KW (1995) Particle Size Distribution of Airborne Dusts Using a Scanning Electron Microscope. Aerosol Science and Technology 23(3):475-478. https://doi.org/10.1080/02786829508965330

[12] Cartwright J (1962) Particle Shape Factors. Annals of Occupational Hygiene 5(3):163-171. https://doi.org/10.1093/annhyg/5.3.163

[13] Bureau International des Poids et Mesures-Joint Committee for Guides in Metrology (2008) Evaluation of measurement data Supplement 1 to the Guide to Expression of Uncertainty in Measurement; Propagation of Distributions Using a Monte Carlo Method. JCGM 100:2008. Available http://www.bipm.org/utils/common/documents/jcgm/JCGM_101_2008_E.pdf. Accessed October 24, 2016 


\section{Journal of Research of the National Institute of Standards and Technology}

About the authors: Robert Fletcher is a physicist in the Surface and Trace Chemical Analysis Group of the Materials Measurement Science Division of the Materials Measurement Laboratory at the NIST. He carries out research in particle metrology to support industry, to produce standards and to produce test materials for trace detection security applications.

Nicholas Ritchie is a physicist the Microscopy and Microanalysis Research Group of the Materials Measurement Science Division of the Materials Measurement Laboratory at the NIST. He specializes in automated particle analysis using scanning electron microscopy and quantitative electron probe microanalysis using energy dispersive $x$-ray spectrometers.

David Bright is a retired NIST research chemist and currently an associate for the Materials Measurement Science Division of the Materials Measurement Laboratory at the NIST. He develops image analysis software that is in the public domain and custom utilities for special image analysis applications.

James Filliben is a statistician in the Statistical Design, Analysis \& Modeling Group of the Statistical Engineering Division of the Information Technology Laboratory at the NIST. He is the creator of the Dataplot statistical analysis software system, and specializes in experiment design and EDA (exploratory data analysis).

William Guthrie is a statistician and the Chief of the Statistical Engineering Division of the Information Technology Laboratory at the NIST. His interests include uncertainty assessment, Bayesian statistics, experimental design and statistical computation.

The National Institute of Standards and Technology is an agency of the U.S. Department of Commerce. 